\title{
Deep Convection Triggering by Boundary Layer Thermals. Part II: Stochastic Triggering Parameterization for the LMDZ GCM
}

\author{
Nicolas Rochetin, JeAn-Yves GrandPeix, AND CATHERINE Rio \\ Laboratoire de Météorologie Dynamique, Paris, France \\ FLEUR COUVREUX \\ CNRM-GAME, Météo-France and CNRS, Toulouse, France
}

(Manuscript received 11 December 2012, in final form 3 September 2013)

\begin{abstract}
This paper presents a stochastic triggering parameterization for deep convection and its implementation in the latest standard version of the Laboratoire de Météorologie Dynamique-Zoom (LMDZ) general circulation model: LMDZ5B. The derivation of the formulation of this parameterization and the justification, based on large-eddy simulation results, for the main hypothesis was proposed in Part I of this study.

Whereas the standard triggering formulation in LMDZ5B relies on the maximum vertical velocity within a mean bulk thermal, the new formulation presented here (i) considers a thermal size distribution instead of a bulk thermal, (ii) provides a statistical lifting energy at cloud base, (iii) proposes a three-step trigger (appearance of clouds, inhibition crossing, and exceeding of a cross-section threshold), and (iv) includes a stochastic component.

Here the complete implementation is presented, with its coupling to the thermal model used to treat shallow convection in LMDZ5B. The parameterization is tested over various cases in a single-column model framework. A sensitivity study to each parameter introduced is also carried out. The impact of the new triggering is then evaluated in the single-column version of LMDZ on several case studies and in full 3D simulations.

It is found that the new triggering (i) delays deep convection triggering, (ii) suppresses it over oceanic trade wind cumulus zones, (iii) increases the low-level cloudiness, and (iv) increases the convective variability. The scale-aware nature of this parameterization is also discussed.
\end{abstract}

\section{Introduction}

In the first paper of this series (Rochetin et al. 2014, hereafter Part I), a stochastic parameterization of deep convection triggering was formally presented. It is based on a statistical analysis of cloudy thermals in a large-eddy simulation (LES) of a moist convection case observed in Niamey (Niger) on 10 July 2006 during the African Monsoon Multidisciplinary Analysis (AMMA) campaign. First, the probability distribution functions (PDFs) of the vertical velocities and of the thermal cross sections (or sizes in the following) at cloud base were determined. Then, assuming deep convection to be triggered by thermals with sizes and maximum vertical velocities exceeding some thresholds, a probability of triggering was

Corresponding author address: Nicolas Rochetin, Laboratoire de Météorologie Dynamique, Boite 99, 4, place Jussieu, F-75252 Paris CEDEX 05, France.

E-mail: nicolas.rochetin@lmd.jussieu.fr determined. The triggering process was then parameterized by using random numbers with a uniform distribution between 0 and 1 and by triggering convection whenever the random number was larger than the no-triggering probability.

The present paper is devoted to an actual implementation of this parameterization in the atmospheric general circulation model (AGCM) Laboratoire de Météorologie Dynamique-Zoom (LMDZ), version 5B (LMDZ5B), and to the assessment of its performance in some case studies and in a global simulation.

In GCMs such as LMDZ5B, where shallow and deep convection are represented by separate parameterizations, the triggering scheme is the part of the model that decides whether moist convection should be treated as purely shallow or as mixed shallow and deep. It acts at every time step, so the triggering scheme decides when deep convection begins and when it ends. Over land, it is thus an important driving process of the diurnal cycle of 
convection and of the frequency of occurrence of deep convection.

This frequency is often considered to be overestimated in GCMs (Bechtold et al. 2004). In LMDZ5B simulations, for instance, convection triggers every day over Niamey during the monsoon season, in contradiction with observations where periods of 2 or 3 days without rain are frequent. In addition, most of the current GCMs tend to predict a diurnal precipitation maximum around noon while satellite observations show a precipitation maximum during midafternoon or evening over land in the tropics (Bechtold et al. 2004; Yang and Slingo 2001). This bias of the simulated diurnal cycle is partly due to the triggering of deep convection occurring too early. Guichard et al. (2004) argue that this is partly correlated with the fact that convective parameterizations do not represent the transient regimes between shallow and deep stages, in which the convective boundary layer gradually deepens and produces significant clouds.

The LMDZ5B general circulation model is one of the few GCMs simulating the maximum of continental precipitation in the late afternoon (Rio et al. 2009). This was achieved by introducing an explicit representation of cloudy boundary layer thermals (Rio and Hourdin 2008) to handle the shallow convection phase and an explicit representation of cold pools generated by the evaporation of convective rainfall (Grandpeix and Lafore 2010) that sustain deep convection until early evening. Boundary layer thermals and cold pools are coupled to the deep convection scheme of Emanuel (1991) by introducing two quantities: the available lifting energy (ALE) and available lifting power (ALP) that are provided at the base of convective towers by both thermals and cold pools. In LMDZ5B, however, the triggering criterion is such that, in practice, deep convection is triggered as soon as cloudy thermals become active and cross the inversion layer, which is the case in trade wind regions or over land in the afternoon. Deep convection can thus still be activated a few hours prematurely or too frequently, inhibiting the development of shallow convection associated with thermals.

The aim of this paper is to present the implementation in LMDZ5B of the triggering parameterization described in Part I and to investigate its influence on the two deficiencies mentioned above: (i) the representation of the shallow phase of convection, in both fair-weather and precipitating conditions, and (ii) the simulation of the variability of rain occurrence, which is particularly key in semiarid regions such as Niamey (Niger). The thermal model used in LMDZ5B computes the properties of a mean bulk thermal. However, this information may not be sufficient to trigger deep convection correctly, as we expect deep convection to be triggered by the largest thermals of a thermal population. This is why the new triggering is no longer based on the properties of a mean thermal but on the properties of a thermal spectrum. The first step consists in determining the thermal distribution characteristics (population and mean size) from the single thermal model's variables. Once this has been done, the triggering parameterization described in Part I may readily be used.

The paper is organized into five parts. The first part presents the model and the different cases investigated. The second part presents the parameterization. In the third part, the parameterization is evaluated through the AMMA case study, and in the fourth part, the sensitivity to the parameters is studied. Finally, the impact of the new triggering parameterization on LMDZ's performances is discussed for various single-column case studies and through 3D simulations.

\section{Data and methodology}

\section{a. The LMDZ single-column model}

We base our work on the single-column model (SCM) part of the GCM LMDZ5B (Hourdin et al. 2013), used to perform climate change simulations for the Intergovernmental Panel on Climate Change (IPCC) Fifth Assessment Report. The model has 39 levels in the vertical, with the grid stretched near the surface (first grid point at $35 \mathrm{~m}$ and eight grid points in the first kilometer) and a mean resolution of $800 \mathrm{~m}$ between 1 and $20 \mathrm{~km}$, with eight grid points over $20 \mathrm{~km}$ (last point at $40 \mathrm{~km}$ ). Emanuel's cumulus parameterization (Emanuel 1991) is used for moist convection. Its statistical parameterization of entrainment was modified by Grandpeix et al. (2004) in order to improve the sensitivity of the simulated deep convection to tropospheric relative humidity. The Emanuel scheme also computes a total cloud water content coupled with a statistical cloud representation, which is based on the computation of a cloud fraction using a lognormal probability density function, as suggested by Bony and Emanuel (2001). The thermodynamic effect of ice is not taken into account in the convective parameterization.

Since the version used for the last IPCC report (2007; Hourdin et al. 2006), the GCM has been updated. The new version of the model is described extensively in Hourdin et al. (2013). As mentioned in the introduction, it includes a mass flux representation of boundary layer thermals (Rio and Hourdin 2008; Rio et al. 2010) combined with the diffusive scheme of Mellor and Yamada (1974). The thermal model uses a bulk entraining thermal approach to compute the properties of a mean thermal representing the set of dry and cloudy boundary layer thermals present in a model grid cell. It provides the height of the cloud base $\left(z_{\mathrm{lcl}}\right)$ and cloud top $\left(z_{\mathrm{top}}\right)$ and the vertical profiles of the mean thermal vertical velocity 
$\left[w_{u}^{\prime}(z)\right]$, thermodynamic properties, and fractional coverage $\left[\alpha_{\text {tot }}(z)\right]$. The new version of LMDZ also includes a representation of cold pools fed by the unsaturated convective downdrafts (Grandpeix and Lafore 2010). The deep convection scheme of Emanuel (1991) has been updated in order to control convection triggering and intensity by subcloud lifting processes, namely, thermals and cold pools, in the following way. The triggering criterion is based on the available lifting energy provided by thermals (subscript BL) and wakes (subscript WK), such that

$$
\mathrm{ALE}=\max \left(\mathrm{ALE}_{\mathrm{WK}}, \mathrm{ALE}_{\mathrm{BL}}\right) .
$$

Deep convection is triggered whenever this energy is sufficient to overcome the inhibition:

$$
\text { ALE }>|\mathrm{CIN}| \text {. }
$$

The closure hypothesis suggested by Grandpeix and Lafore (2010) relates the cloud-base mass flux to the power provided by subcloud processes, the convective inhibition, and the vertical velocity at the level of free convection via

$$
M_{b}=\frac{\mathrm{ALP}_{\mathrm{BL}}+\mathrm{ALP}_{\mathrm{WK}}}{|\mathrm{CIN}|+2 w_{b}^{2}} .
$$

The higher the level of free convection (LFC), the higher the updraft vertical velocity, so $w_{b}$ reads $w_{b}=w_{b, \max } /$ $\left[\left(1+\Delta p /\left(p_{1}-p_{\text {lfc }}\right)\right]\left(\right.\right.$ where $w_{b, \max }=6 \mathrm{~m} \mathrm{~s}^{-1}$ and $\Delta p=$ $500 \mathrm{hPa}$ are tunable parameters). On the other hand, the higher $w_{b}$ is, the more energy is lost to overcome the inhibition, which reduces the convective mass flux at the LFC. A recent study conducted by Rio et al. (2013) stressed the importance of how $w_{b}$ is specified, in this particular coupling, in order to capture both continental and oceanic convection intensity, together with active and suppressed phases of the Madden-Julian oscillation. The contributions of the thermals and wakes to the lifting power $\left(\mathrm{ALP}_{\mathrm{BL}}, \mathrm{ALP}_{\mathrm{WK}}\right)$ are proportional to the thirdorder moment of the thermal vertical velocity $\overline{w^{\prime 3}}$ and cold pool spreading velocity $C^{* 3}$, respectively [see Rio et al. (2009) and Rio et al. (2013) for more details].

\section{b. Triggering: The role of thermals and cold pools in $L M D Z$}

\section{1) THermals}

In the bulk thermal scheme, only the average value of the vertical speed at each level is available for the computation of $\mathrm{ALE}_{\mathrm{BL}, \text { bulk. }}$. Then, since we are looking for a maximum velocity at cloud base, Rio et al. (2009) assume that the maximum vertical speed in the cumulus associated with the bulk thermal is a good estimate of this maximum velocity:

$$
\mathrm{ALE}_{\mathrm{BL}, \mathrm{bulk}}=\frac{1}{2}\left\{\max _{z}\left[w_{u}^{\prime}(z)\right]\right\}^{2} .
$$

This somewhat arbitrary assumption, which has been used successfully in climate simulations (Rio et al. 2013), will be further discussed in section $4 \mathrm{c}$.

\section{2) COLD POOLS}

Another subcloud process coupled to the deep convection is the cold pool mechanism. Cold pools are created by the re-evaporation of rain in the clear air below the anvil, and their height corresponds closely to the cloud base. They ensure that deep convection is maintained during the afternoon through their lifting effect. This lifting energy depends on their kinetic energy (given their spreading velocity $C^{*}$ ), which is assumed equal to their wake potential energy, WAPE $=$ $-g \int_{0}^{h_{w}}\left(\delta \theta_{v} / \overline{\theta_{v}}\right) d z$, where $h_{w}$ is the wake height, $\delta \theta_{v}=$ $\theta_{v \text {,wake }}-\theta_{v \text {,ext }}$ is the positive virtual potential temperature difference between the wake and its environment, and $\overline{\theta_{v}}$ is the grid-scale-averaged virtual potential temperature. The spreading velocity is related to the square root of the potential energy stored by the cold pools $C^{*}=$ $k_{*} \sqrt{2 \mathrm{WAPE}}$ (where $k_{*}=0.33$ is a tunable parameter).

In the model, especially over land surfaces, once deep convection has triggered, the cold pool mechanism largely dominates the boundary layer lifting processes in terms of both triggering ( $\mathrm{ALE} \approx \mathrm{ALE}_{\mathrm{WK}}$ ) and closure $\left(\mathrm{ALP} \approx \mathrm{ALP}_{\mathrm{WK}} ;\right.$ not shown $)$.

\section{c. The four cases investigated}

The parameterization was tested in a single-column framework over four academic case studies. As the variables we focus on are difficult to measure, we take as a reference the high-resolution simulations available for the various cases, which have been evaluated against observations in previous studies.

\section{1) CASES OF AFTERNOON DEEP CONVECTION TRIGGERING}

The AMMA case corresponds to a case of deep convection triggering with an isolated thunderstorm over a semiarid surface at Niamey (Niger) in midafternoon (around 1540 LT; see Lothon et al. 2011) on 10 July 2006. We use Couvreux et al. (2012) as a reference paper, in which several LES runs are displayed and compared with observations. Similarly to the LES, the SCM atmospheric column was forced by surface fluxes (latent and sensible) and by large-scale convergence in accordance with the observations reported that day.

The Atmospheric Radiation Measurement (ARM) Deep case corresponds to an early afternoon deep convection triggering case (around 1300 LT) over the Great Plains of Oklahoma on 27 June 1997. We use Guichard et al. (2004) as a reference paper, in which there are cloud-resolving model (CRM) results that are compared 
with observations. As in the high-resolution models, the SCM atmospheric column was forced on its boundaries with surface fluxes and large-scale advection that have been derived from the observations.

\section{2) CASes of Shallow CONVECTiON WITH NO TRIGGER}

The ARM Shallow is a case of the diurnal cycle of nonprecipitating cumulus clouds over the Oklahoma Great Plains on 21 June 1997 (see Brown et al. 2002). We use Brown et al. (2002) as a reference paper, where several LES results are compared to observations. The SCM surface fluxes and large-scale tendencies were derived from observations (as for the different LES).

The Barbados Oceanographic and Meteorological Experiment (BOMEX) case is a case of trade wind cumulus in a quasi-steady regime over a tropical ocean, on 24 June 1969. We use Siebesma et al. (2003) as a reference paper, where several LES results are compared. The SCM atmospheric column was forced with steady surface fluxes and large-scale advection. Radiative tendencies were also prescribed.

\section{The deep convection stochastic triggering parameterization}

\section{a. Reminder of Part I}

Data from the LES of the AMMA case (semiarid) were analyzed to study the geometric and dynamical properties of the cloudy thermals at the lifting condensation level (LCL) during the transition from shallow to deep convection. The thermal cross-sectional spectrum is composed of two exponential distributions. Type- 1 thermals are topped by the smallest clouds, which are not able to trigger deep convection, and type- 2 thermals are topped by the largest cumulus, which may potentially turn into congestus or cumulonimbus. For these types of clouds, in the first part, we propose a triggering formulation organized into three steps.

A preliminary condition is that the boundary layer must be cloudy to allow deep convection triggering (i.e., only moist thermals are expected to trigger).

The first criterion governs the dynamical transition from a regime in which cumulus clouds cannot reach their LFC [so they stay under the convective inhibition (CIN) layer] to a transient regime where at least some cumulus overshoot the CIN but do not reach the high troposphere. This transition takes place when the statistical maximum kinetic energy provided by boundary layer thermals at cloud base verifies

$$
\mathrm{ALE}_{\mathrm{BL}, \mathrm{stat}}>|\mathrm{CIN}|,
$$

where $A L E_{B L, s t a t}$ is

$$
\mathrm{ALE}_{\mathrm{BL}, \mathrm{stat}}=\frac{1}{2} \mathcal{W}_{\max }^{\prime 2}
$$

in which $\mathcal{W}_{\max }^{\prime}$ is the statistical maximum vertical velocity within the field of thermals:

$$
\mathcal{W}_{\max }^{\prime}=\overline{w_{p}^{\prime}}\left[1+\sqrt{\ln \left\{\frac{\left[\frac{S_{2}}{\check{s}} \ln \left(\frac{N_{2}}{\pi_{t}}\right)\right]^{2}}{2 \pi \pi_{t}^{2}}\right\}-\ln \left(\ln \left\{\frac{\left[\frac{S_{2}}{\check{s}} \ln \left(\frac{N_{2}}{\pi_{t}}\right)\right]^{2}}{2 \pi \pi_{t}^{2}}\right\}\right)}\right]
$$

where $\overline{w_{p}^{\prime}}$ is the spatial average vertical velocity over the thermals at cloud base, $S_{2}$ is the mean cross section of type-2 thermals at cloud base, $N_{2}$ is the corresponding thermal population in the study domain of area $S_{d}, \check{s}$ is an arbitrary draft cross section of reference (here $\check{s}=4 \times$ $\left.10^{4} \mathrm{~m}^{2}\right)$, and $\pi_{t}=\ln (2) \approx 0.7$.

The second criterion governs the sharp transition from the transient regime to the deep convection regime, in which at least one of the overshooting cumulus of the domain reaches the high troposphere, turning into a congestus or a cumulonimbus. For each time step $(t, t+\Delta t)$ of the simulation, a no-triggering probability $\hat{P}_{\Delta t}$ is computed: it is equal to the probability that the cloud-base cross section $S_{\max }$ of the widest thermal does not exceed an arbitrary threshold size $S_{\text {trig: }}$ :

$$
\hat{P}_{\Delta t}=\left\{\left[1-\exp \left(\frac{-S_{\text {trig }}}{S_{2}}\right)\right]^{N_{2}}\right\}^{\Delta t / \tau},
$$


where $\Delta t$ is the model time step, $S_{\text {trig }}$ is the threshold cross section, and $\tau$ is the decorrelation time between cloud scenes. In Part I, it was shown that this parameter ranged from 1000 to $2000 \mathrm{~s}$. The probability that a random realization $S_{\max }$ exceeds $S_{\text {trig }}$ is equal to the probability that a random number $0<\mathcal{R}<1$ exceeds the notriggering probability $\hat{P}_{\Delta t}$.

Hence, in a time period $\Delta t$, the stochastic triggering happens if

$$
\mathcal{R}>\hat{P}_{\Delta t} .
$$

To merge the thresholds given in Eqs. (5) and (9), the effective lifting energy $\mathrm{ALE}_{\mathrm{BL}, \mathrm{eff}}$ can be defined as follows:

$$
\begin{aligned}
& \text { If } \mathcal{R}>\hat{P}_{\Delta t} \text { then } \mathrm{ALE}_{\mathrm{BL}, \mathrm{eff}}=\mathrm{ALE}_{\mathrm{BL}, \mathrm{stat}}, \\
& \mathrm{ALE}_{\mathrm{BL}, \mathrm{eff}}=0 \text { otherwise. }
\end{aligned}
$$

As a result, the deep convection triggering criterion becomes

$$
\mathrm{ALE}>|\mathrm{CIN}| \text { with } \mathrm{ALE}=\max \left(\mathrm{ALE}_{\mathrm{BL}, \mathrm{eff}}, \mathrm{ALE}_{\mathrm{WK}}\right) .
$$

Since the triggering criterion determines whether convection is active or not, it is checked at every time step.

\section{b. Thermal spectrum parameterization}

In this subsection, the type- 2 thermal distribution parameterization is presented. This consists in retrieving the type-2 thermal spectrum characteristics $\left(N_{2}\right.$ and $\left.S_{2}\right)$ from the current LMDZ bulk thermal model (Rio and Hourdin 2008). This parameterization represents the ensemble of dry and cloudy thermals by a single thermal, the properties of which are equal to the grid-cell-averaged properties of the thermal population. The mean thermal is driven by buoyancy. The equations of conservation of mass, momentum, liquid potential temperature, and total water are solved on the vertical by specifying the entrainment and detrainment rates (Rio et al. 2010). The fractional coverage $\alpha_{\text {tot }}$ of the thermal is then deduced from the vertical mass flux and the vertical velocity $w_{u}^{\prime}$. The lifting condensation level $z_{\text {lcl }}$ is deduced from the saturation within the thermal, while the thermal top height $z_{\text {top }}$ corresponds to the height where the vertical velocity of the mean thermal vanishes, representing the maximum cloud-top altitude over the cumulus field. Some hypotheses partially derived from the LES analysis presented in Part I are used to compute the type- 2 thermal spectrum characteristics from the mean bulk thermal properties. (i) The first hypothesis is that the single bulk thermal and the thermal spectrum both cover the same total area $S_{\text {tot }}$ in a given domain $S_{d}$ :

$$
N_{1} S_{1}+N_{2} S_{2}=S_{\text {tot }}=\alpha_{\text {tot }} S_{d} .
$$

Note that the contribution of dry thermals reaching the LCL is not taken into account in this formulation.

(ii) It has been shown in section $4 \mathrm{~b}$ of Part I that the ratio of type- 1 thermal fractional coverage to the whole thermal population fractional coverage is nearly constant and close to $30 \%$ :

$$
\frac{N_{1} S_{1}}{\alpha_{\mathrm{tot}} S_{d}}=\epsilon=0.3 .
$$

(iii) The third hypothesis is also suggested in Part I: it assumes that, for type- 2 thermals, there is a linear relationship between their mean typical size at cloud base $\sqrt{S_{2}}$, the mean cloud depth $\left\langle z_{\text {top }}\right\rangle-\left\langle z_{\text {lcl }}\right\rangle$, and the mean cloud-base altitude $\left\langle z_{\text {lcl }}\right\rangle$ over the thermal population, giving the following quadratic formulation for $S_{2}$ :

$$
S_{2}=\left[a\left(\left\langle z_{\text {top }}\right\rangle-\left\langle z_{\text {lcl }}\right\rangle\right)+b\left\langle z_{\text {lcl }}\right\rangle\right]^{2},
$$

where it has been shown in Part I that the pair $a=1$ and $b=0.3$ gives a correct estimate of $S_{2}$. Equation (14) also provides a physical interpretation of the threshold size $S_{\text {trig. }}$. Let us choose $S_{\text {trig }}=1.2 \times 10^{7} \mathrm{~m}^{2}$ (i.e., a critical diameter $d_{\text {trig }}=3900 \mathrm{~m}$ ), with parameters $a=1$ and $b=0.3$ and an average cloud-base level of $z_{\mathrm{lcl}}=2000 \mathrm{~m}$ for the thermals of the domain. Following Eq. (14), the triggering thermal must reach a cloud-top altitude of $z_{\text {top }}=6880 \mathrm{~m}$, which is higher than the freezing level in most cases. In other words, $S_{\text {trig }}$ approximately corresponds to the size reached by the largest thermal when it exceeds the freezing level. Once $S_{2}$ is determined, the combination of Eqs. (12) and (13) gives

$$
N_{2}=\frac{(1-\epsilon) \alpha_{\text {tot }} S_{d}}{S_{2}} .
$$

(iv) The last assumption identifies the spatial average velocity $\overline{w_{p}^{\prime}}$ over the thermal field with the single thermal velocity $w_{u}^{\prime}$ and identifies the arithmetic average of the LCL altitude $\left\langle z_{\text {lcl }}\right\rangle$ with the single thermal LCL altitude $z_{\mathrm{lcl}}$, that is,

$$
\overline{w_{p}^{\prime}}=w_{u}^{\prime}
$$

and

$$
\left\langle z_{\mathrm{lcl}}\right\rangle=z_{\mathrm{lcl}}
$$


For the cloud top, since the thermal model cloud top $z_{\text {top }}$ corresponds to the top of the highest thermal of the field, we cannot directly relate it to the arithmetic average $\left\langle z_{\text {top }}\right\rangle$. Hence, we consider a coefficient $\alpha$, such that

$$
\left\langle z_{\text {top }}\right\rangle=z_{\text {lcl }}+\alpha\left(z_{\text {top }}-z_{\text {lcl }}\right),
$$

where $\alpha=0.33$ gives a good agreement between LES and LMDZ SCM (not shown). For want of further information, we assume that this $\alpha$ value is universal, a simplification that will deserve attention when more LES simulations are available. This tunable parameter expresses the difference between (i) a statistical maximum cloud top returned by the single thermal representation, which considers a generic thermal in an infinite domain in the horizontal dimension, and (ii) a mean maximum cloud top over a limited number of thermals in a prescribed area (returned by the LES). Ultimately, this parameter may depend (i) on the LES case considered, (ii) on the area of the LES study domain (that can influence the mean value of the maximum cloud top), and (iii) on the boundary layer parameterization in a given GCM.

\section{c. Summary}

\section{1) Algorithm}

At this stage, the deep convection triggering algorithm is entirely determined; the following occur at every discrete time of the simulation.

(i) A preliminary condition is to have cloudy thermals inside the domain.

(ii) If the preliminary condition is verified, Eq. (14), Eq. (15), and parameters $a, b, \epsilon$, and $\alpha$ give the type-2 thermal spectrum characteristics, that is, the pair $N_{2}$ and $S_{2}$

(iii) Equation (16) combined with Eq. (7) and introduced into Eq. (6) gives the maximum kinetic energy $\mathrm{ALE}_{\mathrm{BL} \text {,stat }}$ yielded by the type- 2 thermal at LCL. The first criterion [Eq. (5)] is tested.

(iv) If the first criterion is verified, Eq. (8) is computed and the resulting no-triggering probability $\hat{P}_{\Delta t}$ is compared with a random sample $\mathcal{R}$. The second criterion [Eq. (9)] is tested and Eq. (10) gives the $\mathrm{ALE}_{\mathrm{BL}, \text { eff }}$ (the $\mathrm{ALE}_{\mathrm{BL} \text {,stat }}$ accounting for the deep convection triggering).

Note that, since the present triggering considers thermal statistics instead of a bulk thermal, it is more straightforward to connect it to a multiplume parameterization, rather than a bulk-plume-based parameterization. In such a case, the multiplume parameterization should provide, a priori, most of the variables necessary for computing the no-triggering probability $\hat{P}_{\Delta t}$.

\section{2) PARAMETERS}

The parameterization comprises six parameters divided into two groups. In the first group, "thermal parameters" signifies the set of parameters $a, b, \epsilon$, and $\alpha$ relative to the type- 2 thermal spectrum parameterization [i.e., the pair $N_{2}$ and $S_{2}$ in Eqs. (15) and (14), respectively]. In the second group, "triggering parameters" signifies the set of parameters $S_{\text {trig }}$ and $\tau$ relative to the triggering parameterization [Eq. (8)].

\section{3) Sensitivity to the Domain area $S_{d}$}

The study domain area $S_{d}$ under consideration influences $N_{2}$ through Eq. (15). In a GCM, for instance, $S_{d}$ means the gridcell area, but in a single-column framework $S_{d}$ must be specified. Consequently, for each of the case studies defined in section 2c, we must define a study domain of area $S_{d}$.

For the AMMA case, the size of the domain is assumed equal to the LES performed by Couvreux et al. (2012) and similar to the field campaign area as well (see Lothon et al. 2011): that is, $S_{d, \text { Amma }}=10^{4} \mathrm{~km}^{2}(100 \mathrm{~km} \times$ $100 \mathrm{~km})$. For the ARM Deep and ARM Shallow cases, the arbitrary domain size is $S_{d, \mathrm{Eu}}=6.55 \times 10^{4} \mathrm{~km}^{2}$, consistent with the LES performed by Guichard et al. (2004; which is in two dimensions with a domain length of $250 \mathrm{~km}$ ). For the BOMEX case, the domain size is taken to be close to the field campaign area, which is $S_{d, \mathrm{Bo}}=2.5 \times 10^{5} \mathrm{~km}^{2}(500 \mathrm{~km} \times 500 \mathrm{~km})$ according to Holland (1970). In the rest of the paper, these same reference areas will be kept for each of the case studies.

\section{Parameterization evaluation}

\section{a. Thermal parameterization evaluation on the AMMA case}

In this subsection, the LMDZ boundary layer parameterization is evaluated against mean properties of the thermal field simulated by the LES run on the AMMA case. Since deep convection tends to trigger too early in the SCM, the deep convection scheme was switched off in order to assess the ability of the "unperturbed" boundary layer scheme to capture the transition process, before deep convection onset (i.e., around $1630 \mathrm{LT}$ in the LES).

The SCM was then run on the AMMA case with a $\Delta t=60 \mathrm{~s}$ time step and deep convection switched off. Figure 1 compares LES domain-averaged characteristics of the type- 2 thermals with the SCM single thermal 

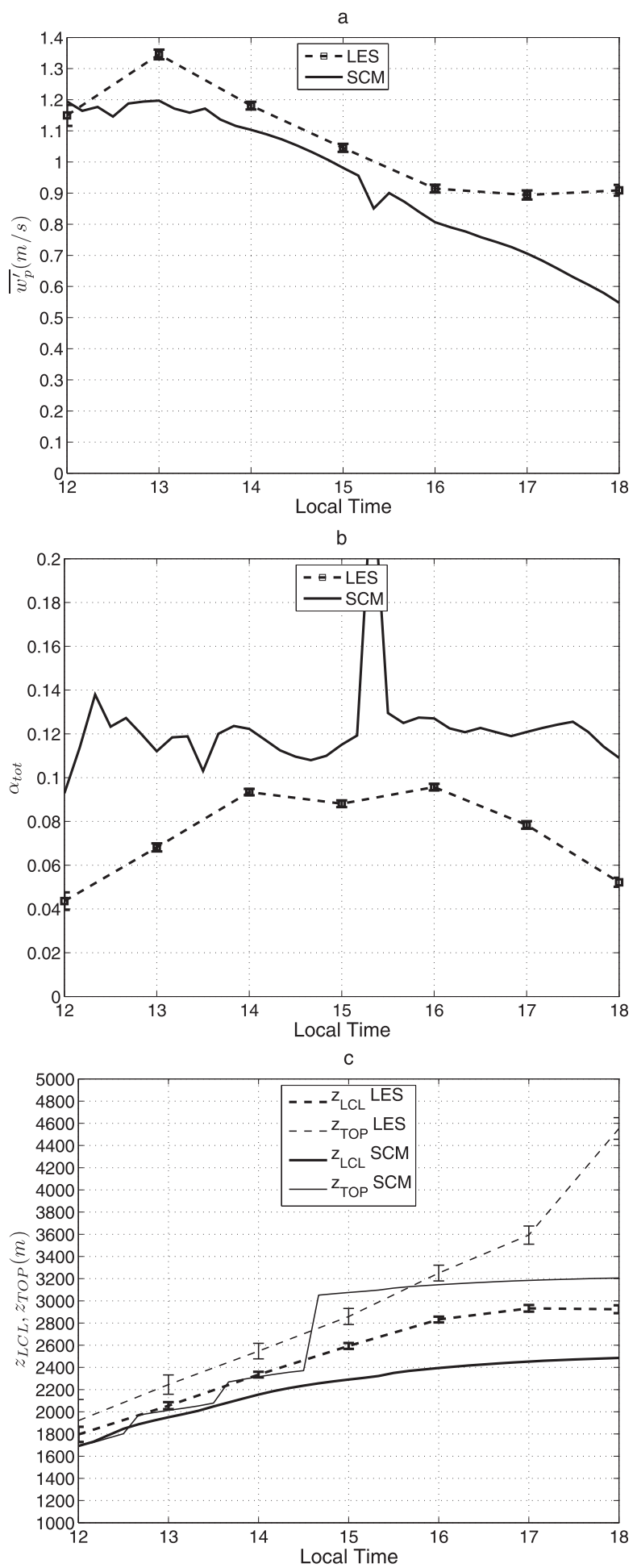

FIG. 1. Time evolution of the characteristics of the thermal field at LCL for the AMMA case: (a) mean vertical velocity over the thermal field $\left\langle\overline{w_{p}^{\prime}}\right\rangle\left(\mathrm{m} \mathrm{s}^{-1}\right)$, computed from the LES (dashed line), against (bulk) thermal velocity $w_{u}^{\prime}$ extracted from the SCM (solid line); (b) as in (a), but for the fractional coverage $\alpha_{\text {tot }}$; and (c) as in (a), but for cloud base (thick lines) and cloud top (thin lines) [see Eq. (18) with $\alpha=0.33$ ]. The SCM's deep convection scheme is switched off and the time step is $\Delta t=60 \mathrm{~s}$. parameterization's outputs at the cloud-base level. Note that, since deep convection only starts around 1630 LT in the LES, which is not represented by the thermal model, the comparison between the LES and the SCM is not relevant from 1630 to $1800 \mathrm{LT}$.

From Fig. 1a, it seems that the SCM and the LES give similar cloud-base velocities, and they both represent the afternoon velocity decrease consistently with the sensible heat flux diminution (not shown).

Figure $1 \mathrm{~b}$ compares the fractional coverage of the resolved versus parameterized thermals at the LCL. The SCM result clearly overestimates the fractional coverage returned by the LES, and it does not really capture the increasing trend from noon to midafternoon and the decreasing trend afterward. This might be due to the fact that the thermal model represents both dry and cloudy thermals. However, the overestimation is largest around noon, when we already know that type- 2 cumuli are not well defined. During the relevant period, that is, from 1330 to $1630 \mathrm{LT}$, the overestimation of the thermal spatial coverage is of the order of 0.03 (i.e., $20 \%$ ), which we shall consider acceptable.

Finally, Fig. 1c compares the cloud base and cloud top of the resolved versus parameterized thermals. The SCM starts to make cumulus around 1330 LT, whereas this happens at around 1100 LT in the LES. The parameterized cumulus also has a 200-400-m lower cloud base and cloud top, and its cloud depth is overestimated from 1500 LT onward.

Therefore, regarding the AMMA case, the LMDZ shallow convection scheme captures the boundary layer and the cumulus cloud deepening simulated by the LES, even though the fractional coverage and cloud depth are overestimated and cumulus onset is delayed. This supports, a posteriori, the assumption we made in Eqs. (16)(18) that the mean properties of the thermal distribution are equivalent to the bulk thermal properties.

It has been shown in Fig. 5 of Part I and in section $3 \mathrm{~b}$ [Eq. (18)] that $a=1, b=0.3, \epsilon=0.3$, and $\alpha=0.33$ is potentially a good set of thermal parameters for representing the time evolution of the thermal spectrum characteristics throughout the transition from shallow to deep convection.

Equation (14) with parameters $a=1$ and $b=0.3, \epsilon=$ 0.3 , and $\alpha=0.33$ is then used in the SCM and the resulting $S_{2}$ and $N_{2}$ are compared with the LES values in Figs. $2 \mathrm{a}$ and $2 \mathrm{~b}$, respectively. The parameterized $S_{2}$ and $N_{2}$ are far from the LES from 1200 to about 1500 LT. The first reason is that the SCM only starts to create cumulus at 1330 LT (see Fig. 1). The other reason has already been discussed in Part I (see Fig. 4 in Part I); the distinction between population 1 and 2 is not clear at the beginning of the transition (from 1100 to $1400 \mathrm{LT}$ ), and the corresponding 
a

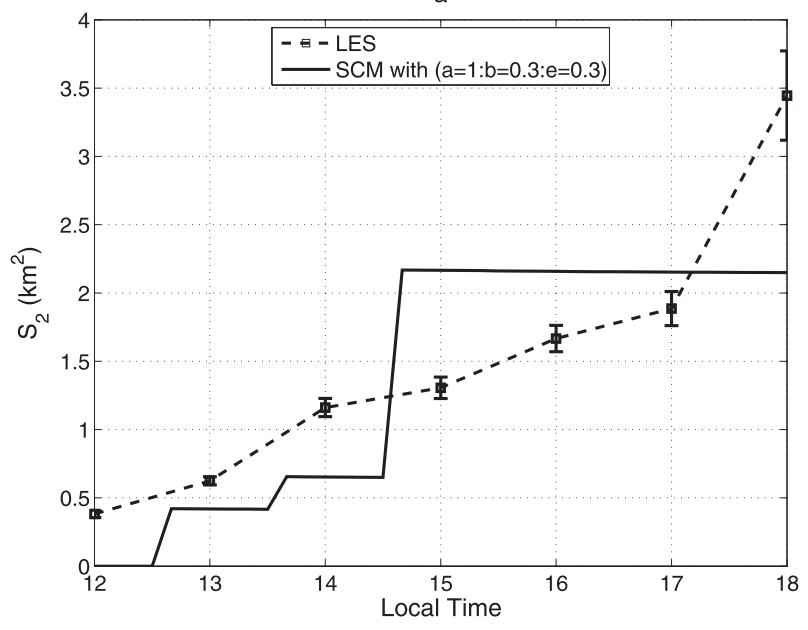

b

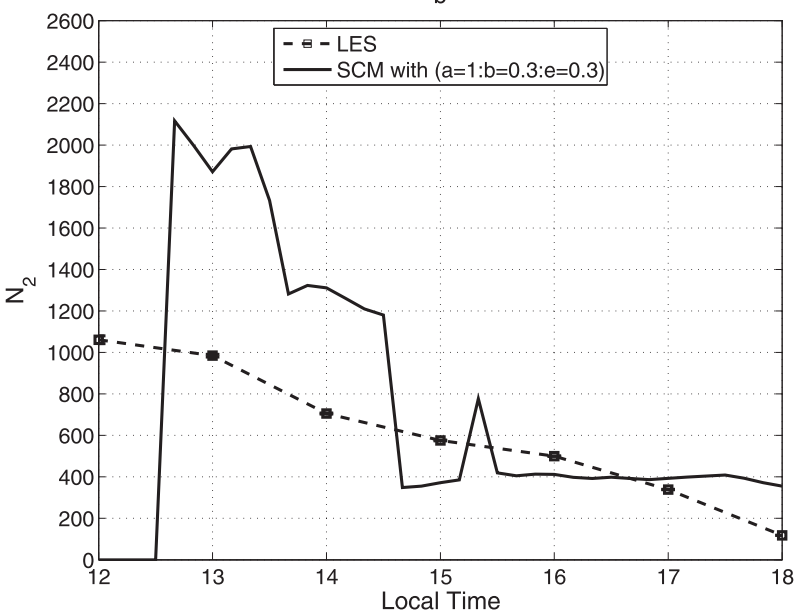

FIG. 2. Time evolution of the characteristics of type-2 thermals: (a) mean cross-sectional area $S_{2}\left(\mathrm{~km}^{2}\right)$ at LCL and (b) population $\mathrm{N}_{2}$, computed from the LES (dashed lines) and extracted from the SCM (solid lines). The large fluctuations of $N_{2}$ (SCM) and $S_{2}$ (SCM) are due to the fluctuation of the simulated cloud top (see Fig. 3c) via Eqs. (15) and (14), respectively. The thermal parameters are $a=1, b=0.3, \epsilon=0.3$, and $\alpha=0.33$ in Eq. (14). The SCM's deep convection scheme is switched off and the time step is $\Delta t=60 \mathrm{~s}$.

thermal cross-section distribution $\mathcal{P}(s)$ resembles a simple exponential more than a sum of exponentials (see Fig. $4 \mathrm{a}$ in Part I). The population 2 distribution becomes more discernible later in the transition process, that is, in that particular case, between 1400 and 1600 LT (see Fig. 4a in Part I). Consequently, the pair $S_{2}$ and $N_{2}$ is strongly correlated with the pair $S_{1}$ and $N_{1}$ before midafternoon (see error bars in Fig. 4c in Part I). Therefore, only the 1400-1600 LT time range is relevant to constrain the thermal parameters. According to Fig. 2, Eqs. (14) and (15) with parameters $a=1, b=0.3, \epsilon=0.3$, and $\alpha=0.33$ give satisfactory results in this time range. In what follows, the set of constrained parameters taken to describe the cross-section spectrum [Eqs. (14), (15)] will then be $a=1, b=0.3, \epsilon=0.3$, and $\alpha=0.33$.

\section{b. Thermal spectrum characteristics over the AMMA, ARM DEEP, BOMEX, and ARM SHALLOW cases}

This subsection studies the distribution of type- 2 thermals' cross sections over the four cases defined in section 2c. The deep convection scheme is switched off for the reason mentioned in the previous subsection.

The model is now run with a time step of $\Delta t=450 \mathrm{~s}$, which is the time step currently used in the standard version of LMDZ5B for climatological runs. The oscillations result from numerical instabilities related to the discretization of the turbulent kinetic energy equation. Parallel work to reduce those instabilities in the full GCM is ongoing. Of course, the oscillations impact the cumulus cloud properties returned by the thermal model and, consequently, the new triggering via the pair $\left(S_{2}\right.$ and $N_{2}$ ) [through Eqs. (14) and (15), respectively]. Nevertheless, we show below that they do not prevent us from making a reasonable assessment of the new triggering parameterization.

The simulated mean cloud-base $\left(z_{\mathrm{lcl}}\right)$ and maximum cloud-top $\left(z_{\text {top }}\right)$ altitudes returned by the bulk thermal model are plotted in Fig. 3. According to Fig. 3, the parameterized boundary layer depth is sensitive to surface moisture and the LCL altitude increases with surface dryness. The semiarid AMMA case exhibits the deepest boundary layer (around $2400 \mathrm{~m}$ ), while the oceanic BOMEX case has the shallowest one (around $400 \mathrm{~m}$ ) and does not show a diurnal cycle for the cloud depth and cloud-base altitudes.

Table 1 gives the time evolution of the characteristics of type-2 thermals, and Fig. 4 shows their corresponding cloud-base diameter $\left(d_{2}=2 \sqrt{S_{2} / \pi}\right)$ and spacing $\left(L_{2}=\right.$ $\sqrt{1 / D_{2}}$, where $D_{2}$ is spatial density $\left.D_{2}=N_{2} / S_{d}\right)$. Over land (AMMA, ARM Deep, and ARM Shallow), according to Eq. (14), the average cross section $S_{2}$ at the LCL increases during the transition phase, in association with the cloud deepening $\left(z_{\text {top }}-z_{\text {lcl }}\right)$ and the cloud-base elevation $\left(z_{\mathrm{lcl}}\right)$ returned by the thermal model (see Fig. 3). Inversely, in accordance with Eq. (15), the total number of type- 2 thermals $N_{2}$ decreases, as does their spatial density $D_{2}$. As $N_{2}$ is inversely proportional to $S_{2}$ in Eq. (15), boundary layer thermal structures become wider and deeper and, consequently, less numerous during the transition in the domain considered. We also learn from Fig. 4 that, throughout the continental transition phase, the thermals and their associated cumulus become more spaced: cloud centers start from $L_{2}=3000 \mathrm{~m}$ spacing and reach about $L_{2}=6000 \mathrm{~m}$ at triggering. 

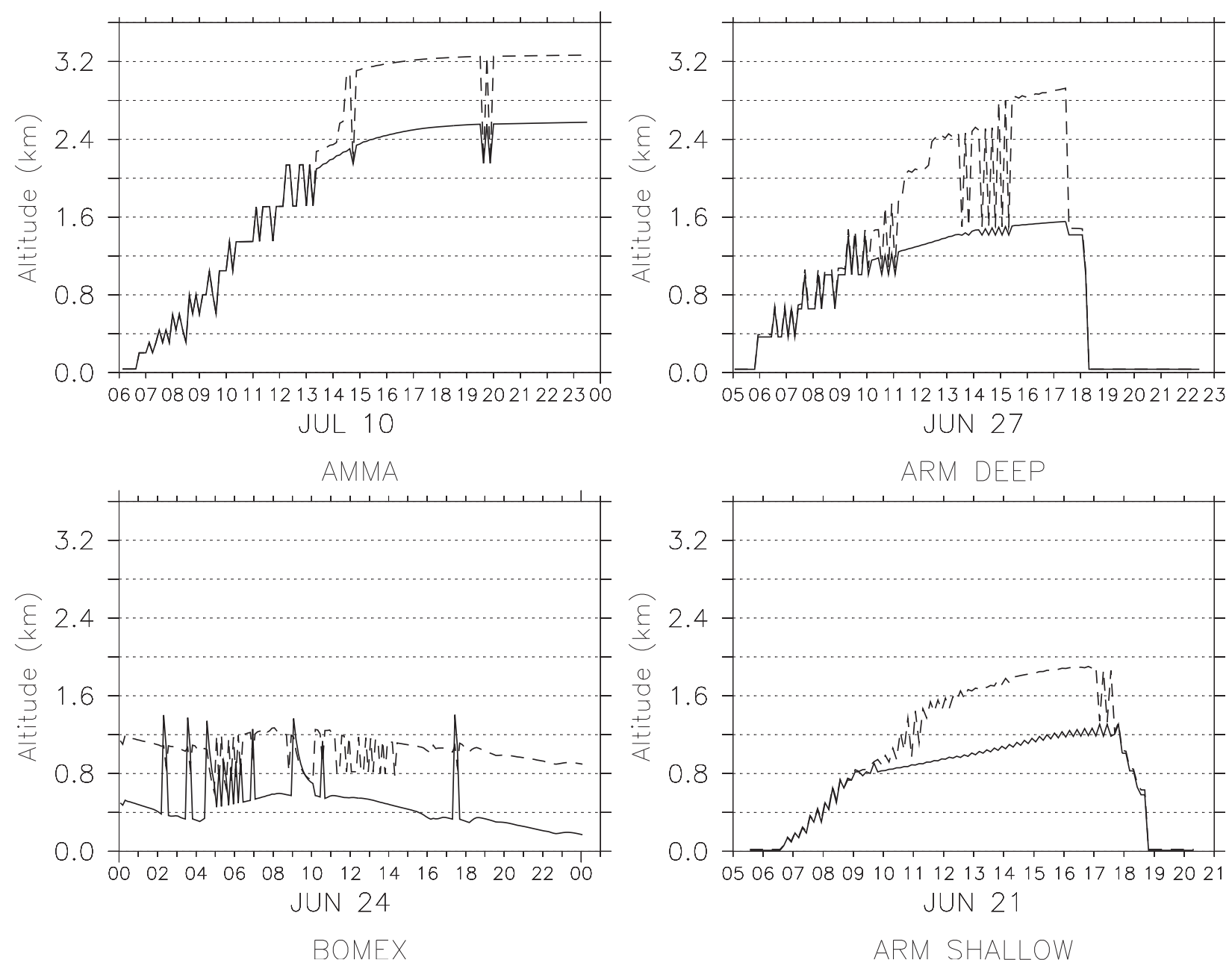

FIG. 3. Time evolution of the cloud-base $z_{\mathrm{lcl}}$ (solid lines) and cloud-top $z_{\text {top }}$ (dashed lines) altitude (km) extracted from the SCM for the (top) (left) AMMA and (right) ARM Deep, and (bottom) (left) BOMEX, and (right) ARM Shallow cases. Fluctuations are sometimes large enough to produce episodic dry boundary layer stages (i.e., the cumulus clouds "disappear" episodically). The SCM's deep convection scheme is switched off and the time step is $\Delta t=450 \mathrm{~s}$. The thermal scheme produces large fluctuations of the cumulus depth when it is run with a $\Delta t=450 \mathrm{~s}$ time step.

Equation (14) is at the core of this parameterization and makes a direct link between the bulk thermal and the spectral thermals formulation. The parameterization is then very sensitive to both the cloud depth $\left(z_{\text {top }}-z_{\text {lcl }}\right)$ and the cloud-base altitude $\left(z_{\mathrm{lcl}}\right)$ simulated by the thermal model. We also recall that Eq. (14) is, of course, very sensitive to the parameters $a$ and $b$, but we assume that the pair $a=1$ and $b=0.3$ is reasonably true.

For the cases in which deep convection triggering has been observed (i.e., AMMA and ARM Deep), Table 1 shows that $S_{2}$ is about twice as large in the AMMA case than in the ARM Deep case at the triggering time (i.e., 1600 LT for AMMA and 1300 LT for ARM Deep). In the oceanic case BOMEX, cloud structures are much more numerous, smaller, and less spaced out (see Table 1 and Fig. 4), resulting in a spatial density $D_{2}$ often exceeding one cloud per square kilometer.

\section{c. Bulk versus statistical $\mathrm{ALE}_{\mathrm{BL}}$}

Figure 5 plots $\mathrm{ALE}_{\mathrm{BL}, \text { bulk }}$ and $\mathrm{ALE}_{\mathrm{BL}, \text { stat }}$ [Eq. (6)] against $\mathrm{CIN}$ for all cases. Let us focus first on continental cases. Depending on the case, $\mathrm{ALE}_{\mathrm{BL} \text {,stat }}$ exceeds $|\mathrm{CIN}|$ either before or after $A_{L E} E_{B L, b u l k}$. For instance, in the AMMA case, the statistical ALE BL $_{\mathrm{BL}}$ overcomes the inhibition at around $1315 \mathrm{LT}, 1 \mathrm{~h}$ after the bulk one. But, in the ARM Deep case, the bulk ALE $_{\mathrm{BL}}$ crosses the inhibition $1 \mathrm{~h}$ later than the statistical one (i.e., around $1115 \mathrm{LT}$ ), and both $\mathrm{ALE}_{\mathrm{BL} \text {,bulk }}$ and $\mathrm{ALE}_{\mathrm{BL} \text {,stat }}$ trigger simultaneously (around 1100 LT) in the ARM Shallow case. Looking at those three continental cases, the effects of the $A L E_{B L, s t a t}$ and $A L E_{B L, b u l k}$ triggers appear comparable. This suggests that the bulk thermal model together with the bulk ALE given by Eq. (4) gives a good first-order estimation for treating continental convection. 
TABLE 1. Time evolution of the characteristics of type-2 thermals simulated by the SCM run on the AMMA, ARM Deep, BOMEX, and ARM Shallow cases: mean cross section $S_{2}\left(\mathrm{~km}^{2}\right)$, population $N_{2}$, and spatial density $D_{2}\left(\mathrm{~km}^{-2}\right)$. The zero values correspond to dry boundary layer stages. The thermal parameters in Eq. (14) are $a=1, b=0.3, \epsilon=0.3$, and $\alpha=0.33$. The SCM's deep convection scheme is switched off and the time step is $\Delta t=450 \mathrm{~s}$.

\begin{tabular}{|c|c|c|c|c|c|c|c|c|c|c|c|c|}
\hline \multirow[b]{2}{*}{ Local time } & \multicolumn{3}{|c|}{ AMMA } & \multicolumn{3}{|c|}{ ARM Deep } & \multicolumn{3}{|c|}{ BOMEX } & \multicolumn{3}{|c|}{ ARM Shallow } \\
\hline & $S_{2}$ & $N_{2}$ & $D_{2}$ & $S_{2}$ & $N_{2}$ & $D_{2}$ & $S_{2}$ & $N_{2}$ & $D_{2}$ & $S_{2}$ & $N_{2}$ & $D_{2}$ \\
\hline 0800 & 0 & 0 & 0 & 0 & 0 & 0 & 0.34 & 76406 & 0.306 & 0 & 0 & 0 \\
\hline 0900 & 0 & 0 & 0 & 0 & 0 & 0 & 0.29 & 348299 & 1.393 & 0 & 0 & 0 \\
\hline 1000 & 0 & 0 & 0 & 0 & 0 & 0 & 0 & 0 & 0 & 0.16 & 18346 & 0.280 \\
\hline 1100 & 0 & 0 & 0 & 0.53 & 15325 & 0.234 & 0.27 & 277350 & 1.109 & 0.64 & 3750 & 0.057 \\
\hline 1200 & 0 & 0 & 0 & 0.8 & 8667 & 0.132 & 0.25 & 325149 & 1.301 & 0.71 & 3100 & 0.047 \\
\hline 1300 & 0 & 0 & 0 & 1.14 & 5136 & 0.078 & 0.17 & 653996 & 2.616 & 0.79 & 2278 & 0.034 \\
\hline 1400 & 0.65 & 1842 & 0.184 & 1.14 & 5934 & 0.091 & 0.24 & 332943 & 1.331 & 0.88 & 1743 & 0.027 \\
\hline 1500 & 2.16 & 513 & 0.051 & 1.56 & 4099 & 0.063 & 0.33 & 213492 & 0.854 & 0.87 & 1586 & 0.024 \\
\hline 1600 & 2.15 & 332 & 0.033 & 1.56 & 3901 & 0.060 & 0.36 & 322005 & 1.288 & 0.87 & 1550 & 0.024 \\
\hline 1700 & 2.15 & 304 & 0.030 & 1.56 & 3001 & 0.046 & 0.36 & 323064 & 1.292 & 0.87 & 1699 & 0.026 \\
\hline 1800 & 2.15 & 265 & 0.027 & 0 & 0 & 0 & 0.27 & 170473 & 0.682 & 0 & 0 & 0 \\
\hline
\end{tabular}

Looking at $\mathrm{ALE}_{\mathrm{BL} \text {,stat }}$ only, large differences can be seen in all cases in Fig. 5. As shown in Eq. (6), $\mathrm{ALE}_{\mathrm{BL}, \mathrm{stat}}$ is mostly related to the thermal vertical velocity at the LCL $w_{u}^{\prime}$, which depends on both buoyancy and depth of the boundary layer. In all the continental cases, $\mathrm{ALE}_{\mathrm{BL} \text {,stat }}$ overcomes the CIN and a transient regime of several hours occurs during the afternoon, suggesting that strong updrafts feed cumulus that have exceeded their level of free convection. Regarding Fig. 5, transition starts at $1315 \mathrm{LT}$ for the AMMA case, at $1000 \mathrm{LT}$ for the ARM Deep case, and at 1100 LT for ARM Shallow case, which is consistent with LES outputs. It is worth noting that the very large $A L E_{B L, s t a t}$ in ARM Shallow is explained by both the very large $w_{u}^{\prime}$ at LCL $\left(w_{u}^{\prime} \approx 2.2 \mathrm{~m} \mathrm{~s}^{-1}\right.$, not shown) and the large number of type- 2 clouds $N_{2}$ (see Table 1 ).

In the oceanic case (i.e., BOMEX), differences between $\mathrm{ALE}_{\mathrm{BL} \text {,bulk }}$ and $\mathrm{ALE}_{\mathrm{BL} \text {,stat }}$ are more prominent. In this case $A L E_{B L, s t a t}$ stays below the CIN most of the time, but $\mathrm{ALE}_{\mathrm{BL} \text {,bulk }}$ is almost always higher than the $|\mathrm{CIN}|$. Indeed, the thermal vertical velocity at LCL in the BOMEX case $\left(w_{u}^{\prime} \approx 0.4 \mathrm{~m} \mathrm{~s}^{-1}\right)$ is half that in the AMMA case $\left(w_{u}^{\prime} \approx 1 \mathrm{~m} \mathrm{~s}^{-1}\right)$, resulting in a correspondingly low value of $\mathcal{W}_{\max }^{\prime}$ [see Eq. (7)]. Therefore, the statistical $\mathrm{ALE}_{\mathrm{BL} \text {,stat }}$ cancels deep convection. Knowing that the standard version of LMDZ5B tends to produce some convective rainfall over oceanic, trade wind cumulus zones (e.g., BOMEX), this result is of key importance.

To sum up, the main differences between ALE $_{\mathrm{BL}, \text { bulk }}$ and $A L E_{B L, s t a t}$ come from the reference altitude chosen for the ALE computation. For ALE $\mathrm{BL}_{\mathrm{BL}, \text { bulk, }}$, the reference altitude corresponds to the bulk thermal maximum velocity, either dry or cloudy. For $\mathrm{ALE}_{\mathrm{BL}, \mathrm{stat}}$, it is the bulk thermal cloud base (so it must be cloudy).

The first consequence is that the bulk ALE can be positive, even if the bulk thermal is dry. That is why, in
Fig. 5, the ALE $E_{B L, b u l k}$ is positive early in the morning, despite there being no cloud in the domain, and exceeds the inhibition an hour earlier than the $\mathrm{ALE}_{\mathrm{BL}, \mathrm{stat}}$.

The second point is that the bulk thermal model often returns a local minimum of the vertical velocity near the cumulus base and a maximum near cumulus top. The ratio between the maximum and the minimum velocity depends on the thermodynamic properties of the bulk thermal (and ultimately on the bulk cumulus depth) and may vary greatly from one case to another. Consequently, $A L E_{B L, s t a t}$ can be either larger or smaller than $\mathrm{ALE}_{\mathrm{BL}, \text { bulk}}$.

\section{d. An example of stochastic triggering in the AMMA case}

The SCM was run in the AMMA case, with the new triggering for deep convection, with the thermal parameters $a=1, b=0.3, \epsilon=0.3$, and $\alpha=0.33$ and the triggering parameters $S_{\text {trig }}=12 \mathrm{~km}^{2}$ and $\tau=1000 \mathrm{~s}$. Deep convection was also switched on and the time step was kept at $\Delta t=450 \mathrm{~s}$. Figure 6 shows the deep convection triggering scenario.

The thermal model does not create any cumulus (not shown) before $1330 \mathrm{LT}$, explaining why $\mathrm{ALE}_{\mathrm{BL} \text {,stat }}=0$. After 1330 LT, some clouds appear and, a short time later, the dynamical criterion is reached [Eq. (5)]. The transient regime starts, which means that at least one cloud hosts an updraft with kinetic energy exceeding the CIN. A random generator was run and returned a random sample $\mathcal{R}$ (between 0 and 1 ) at every time step, which could be compared with a no-triggering probability $\hat{P}_{\Delta t}$ computed over the time step period $\Delta t$. During the next hour, the boundary layer and the surrounding clouds deepened (not shown), and consequently, $S_{2}$ 

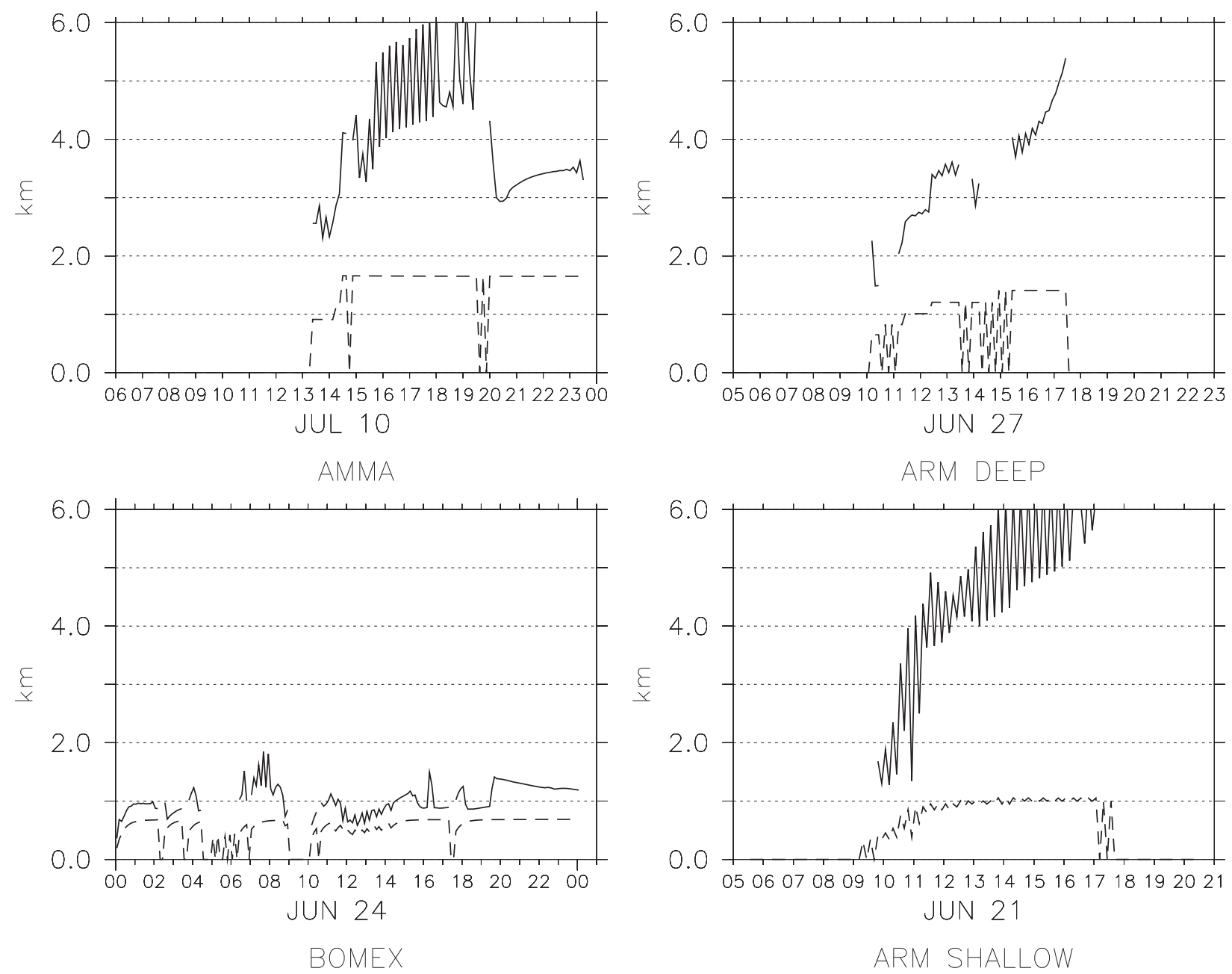

FIG. 4. As in Fig. 3, but for the mean cloud-base diameter $d_{2}$ (dashed lines; km) and spacing $L_{2}$ (solid lines; km) of type-2 thermals at cloud base. The large fluctuations associated with zero values for $d_{2}$ and $L_{2}$ are due to episodic dry boundary layer stages created by the SCM. The thermal parameters are $a=1, b=0.3, \epsilon=0.3$, and $\alpha=0.33$. The SCM's deep convection scheme is switched off and the time step is $\Delta t=450 \mathrm{~s}$.

increased and $N_{2}$ decreased, resulting in a slight decrease of $\hat{P}_{\Delta t}$. As long as the geometrical criterion remains unverified [Eq. (9)], $\mathrm{ALE}_{\mathrm{BL} \text {,eff }}=0$ and deep convection does not trigger [because Eq. (11) is not verified]. Around 1430 LT, a first random realization $\mathcal{R}$ finally exceeds $\hat{P}_{\Delta t}$, the geometrical threshold is reached [Eq. (9)], and deep convection is triggered. Later in the afternoon, the condition $\mathcal{R}>\hat{P}_{\Delta t}$ occurs another seven times. However, a short time after the deep convection onset, the rain re-evaporation produces unsaturated downdrafts and cold pools, which ensures deep convection triggering later on (not shown). The result is then, for that particular run, a deep convection triggering delayed by $1.25 \mathrm{~h}$ as compared with the standard parameterization, which triggers about 1315 LT (not shown).

\section{Triggering-parameter sensitivity experiments with the SCM}

\section{a. Integrated triggering probability $\mathcal{P}_{\Delta t}$}

The main objective of this section is to explore the sensitivity of the new parameterization to the triggering parameters $S_{\text {trig }}$ and $\tau$ and the domain area $S_{d}$ in four distinct cases. The aim is to test the sensitivity of the diurnal cycle of the trigger probability to every set of parameters. Here we do not test the sensitivity to the thermal parameters, as they have been fitted using largeeddy simulations results.

A way to test it is to run a large number of simulations for every set of parameters and to retrieve the triggering time histogram, but this is quite tedious and can be avoided. An alternative way is to run only one simulation for each 


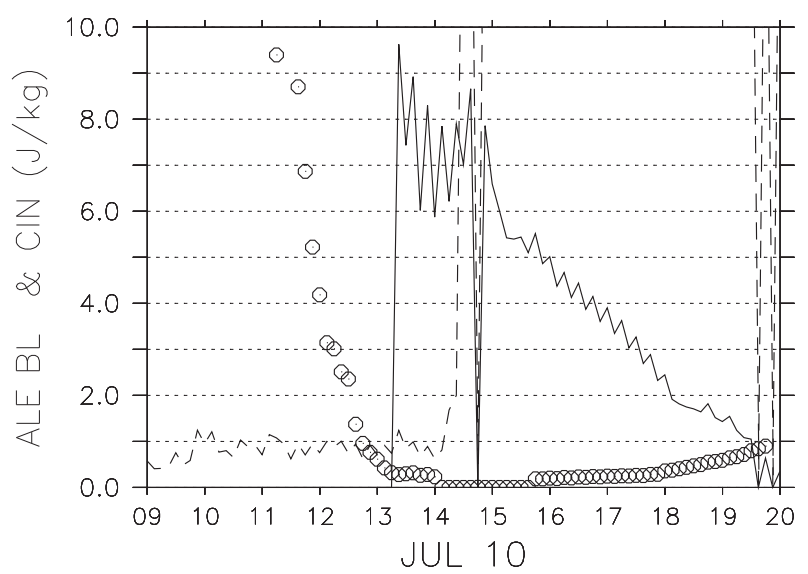

AMMA

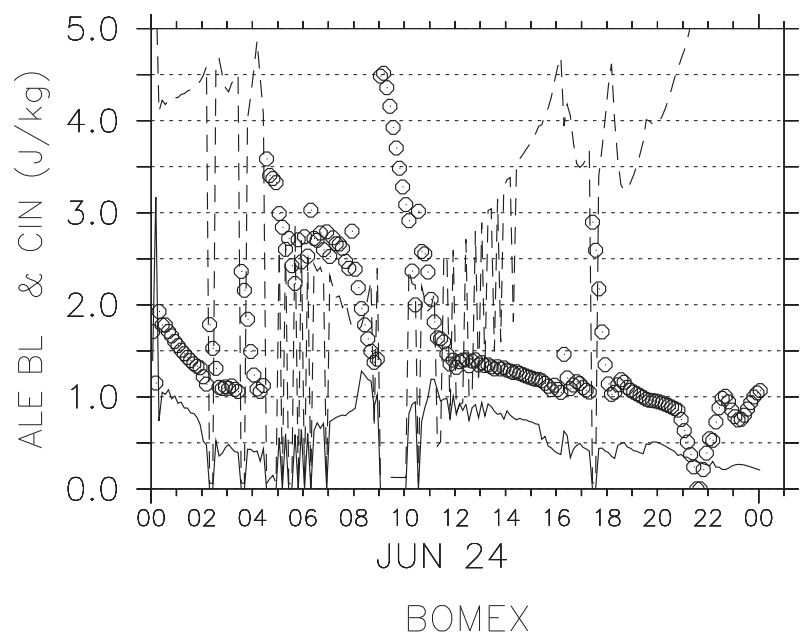

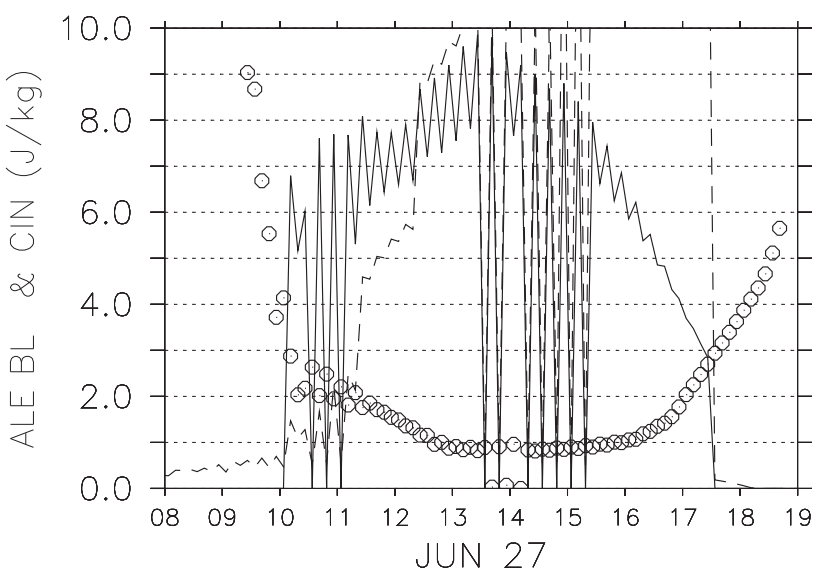

ARM DEEP

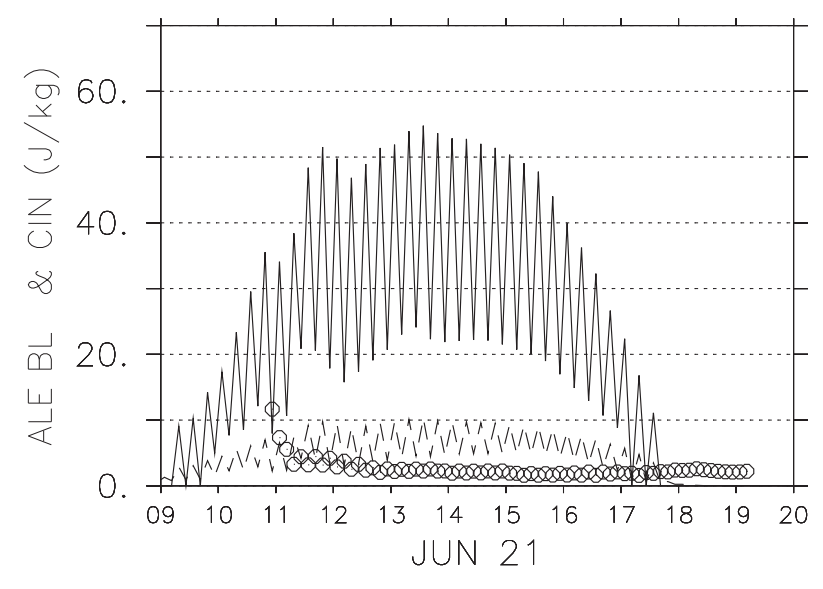

ARM SHALLOW

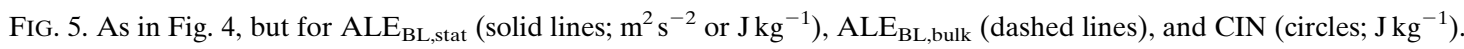

case, with the convection scheme switched off. If deep convection triggering is cancelled, the thermal model produces a unique (deterministic) time series of $\left(S_{2}\right.$ and $\mathrm{N}_{2}$ ) pairs [Eqs. (14), (15)]. Then, we want to compute the probability that convection triggers in a time interval $\left(t_{0}\right.$, $t_{n}$ ) made up of $n$ time steps of length $\Delta t$. We shall call this probability the integrated triggering probability $\mathcal{P}_{\Delta t}$. To that end, we first compute the no-triggering probability, which is the product of the no-triggering probabilities of each time step:

$$
\hat{\mathcal{P}}_{\Delta t}\left(t_{n}\right)=\prod_{k=0}^{n-1} \hat{P}_{\Delta t}\left(t_{k}\right)
$$

Then, the integrated triggering probability reads

$$
\mathcal{P}_{\Delta t}\left(t_{n}\right)=1-\prod_{k=0}^{n-1} \hat{P}_{\Delta t}\left(t_{k}\right) .
$$

Thus, from a single no-triggering scenario, we can deduce the time series of the integrated triggering probability $\mathcal{P}_{\Delta t}$ corresponding to a particular set of free parameters $S_{\text {trig }}$ and $\tau$. Therefore, the triggering sensitivity study requires only one convection-free simulation per case and per parameter configuration.

\section{b. Sensitivity to the threshold cross section $S_{\text {trig }}$}

The first experiment concerns the triggering parame-

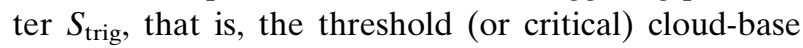
cross section above which the cumulus cloud becomes a congestus or a cumulonimbus (deep convective cloud). The thermal parameters are set to $a=1, b=0.3, \epsilon=0.3$, and $\alpha=0.33$ and the decorrelation time is set to $\tau=$ 1000 s. For each case study, the domain area considered is the reference area (mentioned in section 3c).

Figure 7 displays the integrated trigger probability $\mathcal{P}_{\Delta t}$ for the range $S_{\text {trig }}=10,12,15,18$, and $20 \mathrm{~km}^{2}$ (i.e., a critical diameter $D_{\text {trig }}=3570,3910,4370,4790$, and 

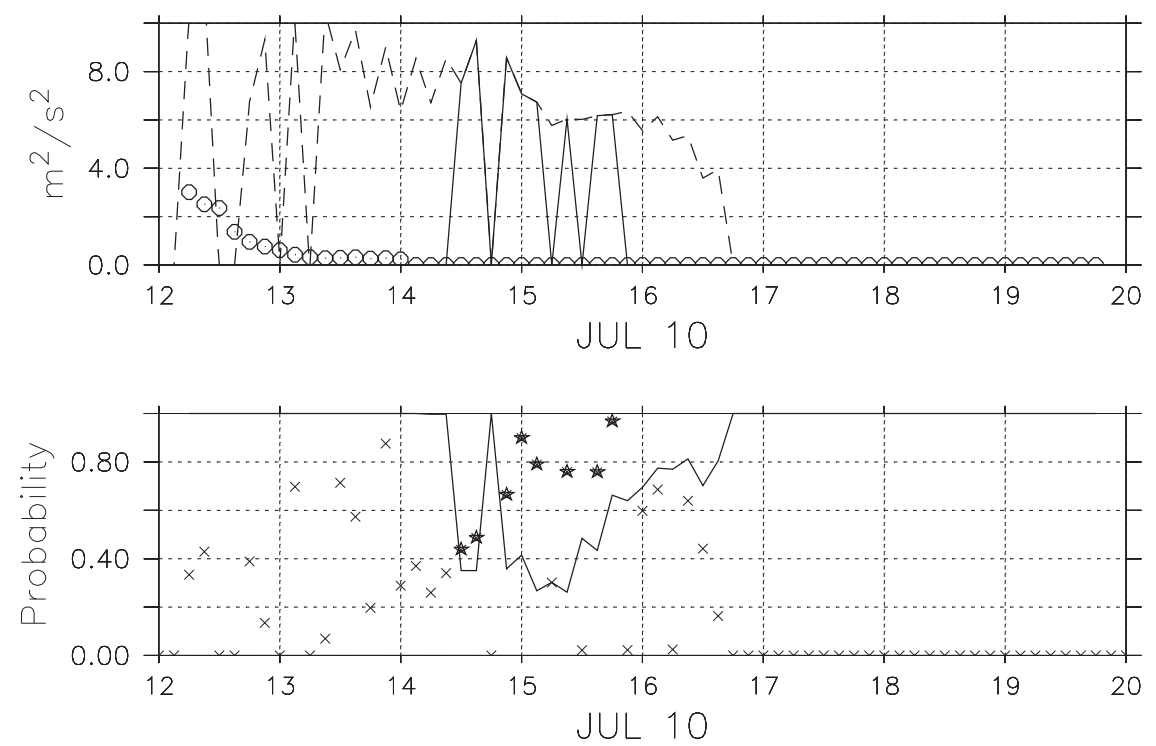

FIG. 6. Time evolution of (top) $|\mathrm{CIN}|$ (circles; $\mathrm{J} \mathrm{kg}^{-1}$ ), $\mathrm{ALE}_{\mathrm{BL} \text {,stat }}$ (dashed line; $\mathrm{m}^{2} \mathrm{~s}^{-2}$ or J kg$^{-1}$ ), and $\mathrm{ALE}_{\mathrm{BL} \text {,eff }}$ (solid line). (bottom) Probability of no-triggering $\hat{P}_{\Delta t}$ (solid line), random sample $\mathcal{R}$ (crosses), and $\mathcal{R}$ at triggering (bold stars) simulated by the SCM for the AMMA case. The thermal parameters are $a=1, b=0.3, \epsilon=0.3$, and $\alpha=0.33$ and the triggering parameters are $S_{\text {trig }}=12 \mathrm{~km}^{2}$ and $\tau=1000 \mathrm{~s}$. The SCM's deep convection scheme is activated and the time step is $\Delta t=450 \mathrm{~s}$.

$5000 \mathrm{~m})$. The expected sigmoid shape occurs in several instances, but not always. Some curves show several steps or ramps (these peculiarities are commented below). The integrated triggering probability decreases when $S_{\text {trig }}$ increases, in agreement with Eq. (8), which shows that the no-triggering probability $\hat{P}_{\Delta t}$ is an increasing function of $S_{\text {trig. }}$. For small values of $S_{\text {trig }}$, the probability of triggering increases fast with cloud deepening $\left(z_{\text {top }}-z_{\text {lcl }}\right)$ and cloud-base elevation $\left(z_{\text {lcl }}\right)$. Hence, the triggering diurnal cycle is shifted earlier and is more peaked (because cumulative probabilities are bounded by one, an increased probability of early triggering automatically corresponds to a decreased probability later). When $S_{\text {trig }}$ increases, the diurnal cycle of triggering probability is more spread through the afternoon, and the total probability $\left[\mathcal{P}_{\Delta t}\left(t_{\text {final }}\right)\right]$ is also reduced. For example, the total triggering probability is $87 \%$ for $S_{\text {trig }}=18 \mathrm{~km}^{2}$ and $55 \%$ for $S_{\text {trig }}=20 \mathrm{~km}^{2}$ in the AMMA case, and even falls to $8 \%$ for $S_{\text {trig }}=20 \mathrm{~km}^{2}$ in the ARM Deep case.

It can be observed that, for intermediate values of $S_{\text {trig }}$, the ARM Deep case exhibits a double-peaked diurnal cycle of $\mathcal{P}_{\Delta t}$, with two distinct time periods favorable for triggering and a "suppressed" period in between. This can be understood by considering Fig. 3 (top-right panel). Between 1300 and 1530 LT, the thermal model exhibits large oscillations, and even some dry stages, during which $\hat{P}_{\Delta t}=1$.

The AMMA case also looks more favorable to triggering than the ARM Deep case, even though both have a similar cloud depth. This is because the average cross section $S_{2}$ is an increasing function of the cloud-base height $z_{\text {lcl }}$ [see Eq. (14)], and $z_{\text {lcl }}$ is twice as high in the AMMA case. The ARM Shallow case only triggers for the smallest values of $S_{\text {trig. }}$. Finally, as mentioned in the previous subsection, the BOMEX simulation has no chance of triggering because $\mathrm{ALE}_{\mathrm{BL} \text {,stat }}<|\mathrm{CIN}|$ during almost all the simulation.

The first conclusion is that the cloud-base altitude $z_{\text {lcl }}$ plays a key role in controlling the diurnal cycle of triggering probability, especially through Eqs. (14) and (8). This parameter is actually the main discriminatory factor between cases, since, in all these cases, the parameterized mean cloud depths are very close (around $600 \mathrm{~m}$ ), but the integrated triggering probability increases with the cloud-base height. Over wet soils or oceans, since the cloud base is low, the vertical extension of the cloud must be greater than over dry surfaces if deep convection is to be triggered. The transition period is then longer. Note that this result is consistent with the observational study performed by Hohenegger and Stevens (2013). Even though overshooting cumulus are present in the domain, their average cross section $S_{2}$ is small because of a low $z_{\mathrm{lcl}}$, and the no-triggering probability $\hat{P}_{\Delta t}$ [Eq. (8)] stays very close to 1 . Over dry soils, clouds appear later but, since the boundary layer is deeper, thermal structures and corresponding cloud bases are wider (following the hypothesis of a fixed aspect ratio for boundary layer thermal cells). This results 

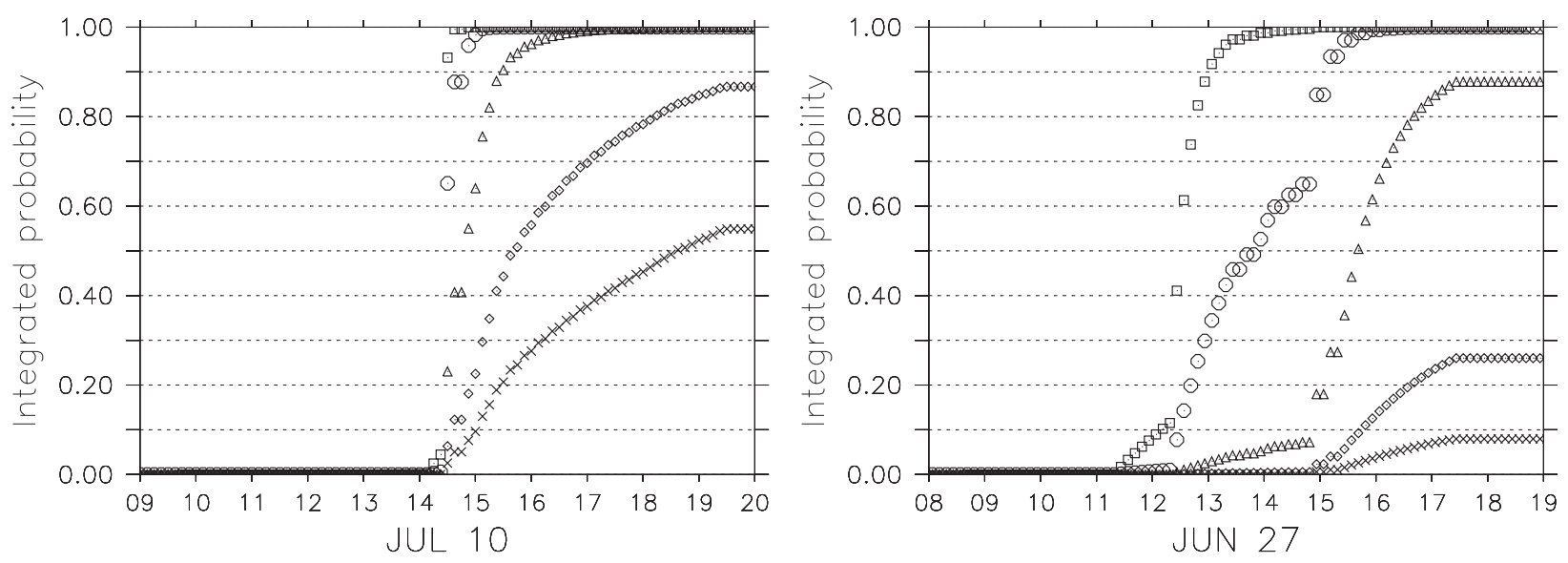

AMMA
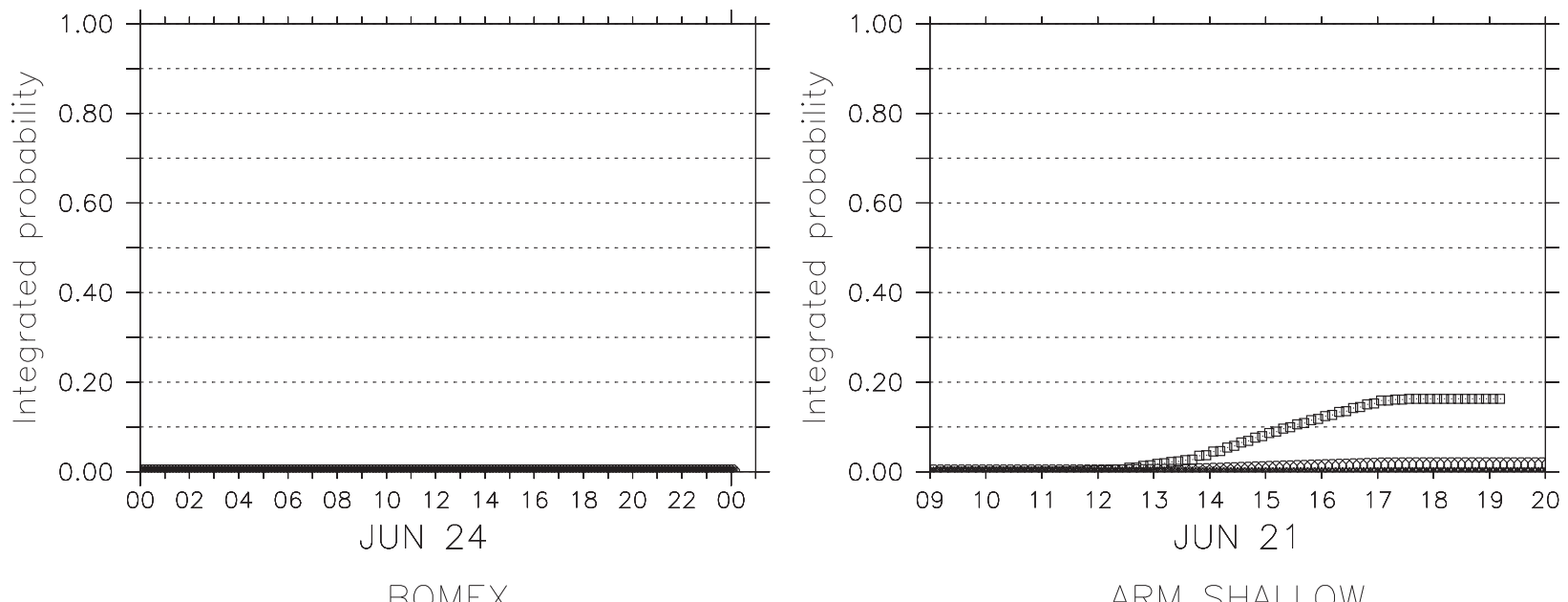

BOMEX

FIG. 7. As in Fig. 4, but for integrated triggering probability $\mathcal{P}_{\Delta t}$ for the threshold cross sections $S_{\text {trig }}=10$ (squares), 12 (circles), 15 (triangles), 18 (diamonds), and $20 \mathrm{~km}^{2}$ (crosses) cases. The triggering decorrelation time is $\tau=1000 \mathrm{~s}$.

in a shorter transition period with cumuli needing less vertical extension to trigger deep convection.

The second point is that an intermediate stage appears between shallow and deep regimes. For instance, when $S_{\text {trig }}=20 \mathrm{~km}^{2}$, the total triggering probability for the AMMA case is $55 \%$ while it is $8 \%$ for the ARM Deep case. Thus, even though large-scale and surface conditions still play a key role in the triggering (e.g., cancel it in BOMEX), the new parameterization allows the model to have an intermediate stage, in which similar conditions can give different results (triggering or no triggering). When the threshold cross section $S_{\text {trig }}$ increases, this intermediate stage lasts longer.

\section{c. Sensitivity to the decorrelation interval $\tau$}

The sensitivity of the probabilistic triggering diurnal cycle to the triggering decorrelation time $\tau$ is now approached. The chosen range of $\tau$ values (from 1000 to $1800 \mathrm{~s}$ ) is assumed to enclose all possible lifetimes of a type- 2 cumulus cloud (see Part I), and the threshold cross section is set to $S_{\text {trig }}=12 \mathrm{~km}^{2}$.

From Eq. (8), $\hat{P}_{\Delta t}$ is an increasing function of $\tau$. An increase in the time period between two independent cloud scenes means a decrease by the same factor of the chance of triggering in a given period $\Delta t$. Actually, Fig. 8 shows that an increase in $\tau$ reduces the integrated triggering probability (thus delaying the triggering). This is more obvious in the ARM Deep and the ARM Shallow cases than in the AMMA case because, in the AMMA case, all the triggering scenarios are concentrated in a very short period of time when $S_{\text {trig }}=12 \mathrm{~km}^{2}$ (i.e., between 1415 and 1500 LT; see Fig. 7, top-left panel).

\section{d. Sensitivity to the domain area $S_{d}$ and scale awareness}

The model's sensitivity to the reference domain area $S_{d}$ is now studied with parameters $S_{\text {trig }}=12 \mathrm{~km}^{2}$ and $\tau=$ $1200 \mathrm{~s}$. Note that, even though we chose $\tau=1000 \mathrm{~s}$ in the 

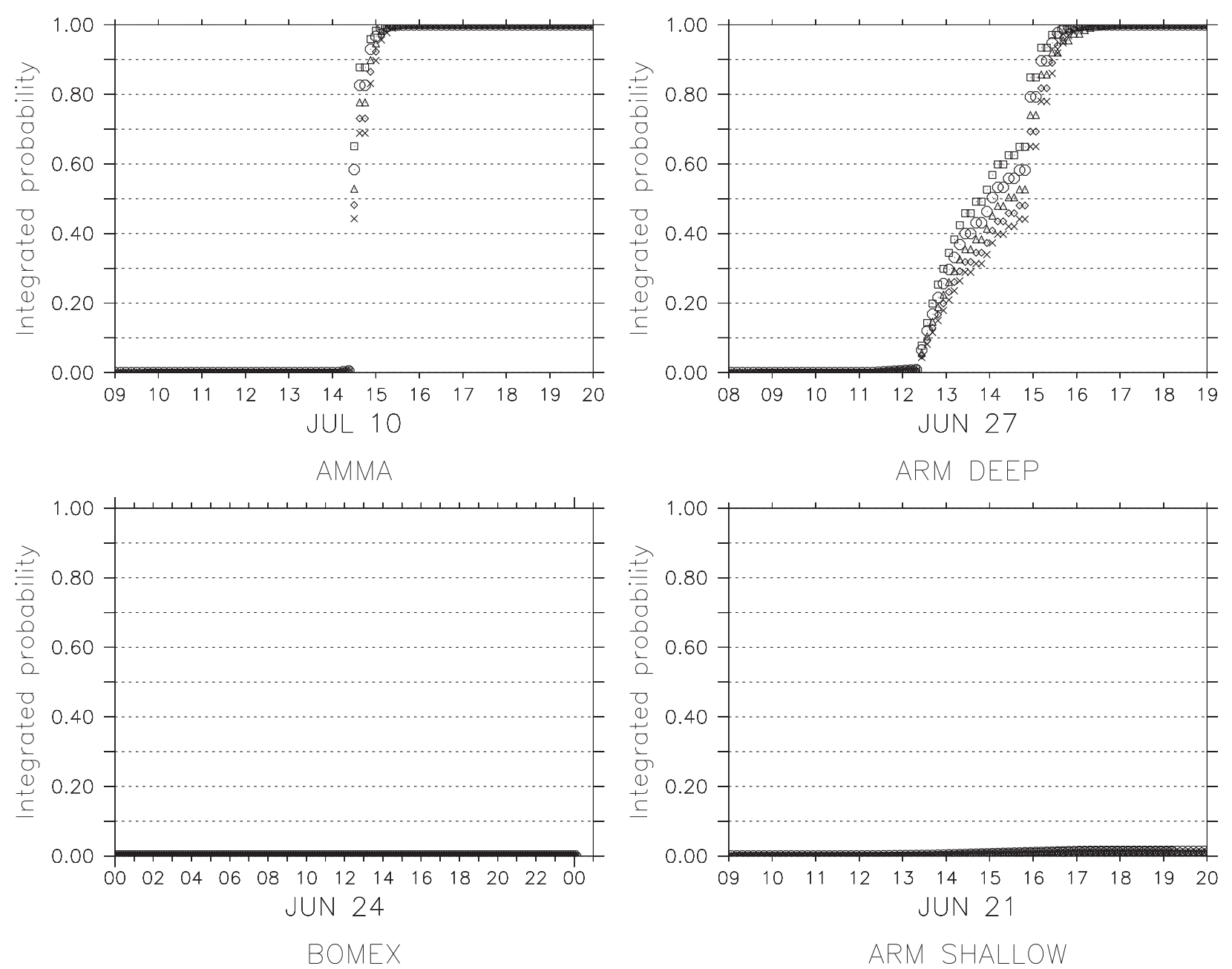

FIG. 8. As in Fig. 7, but for the decorrelation intervals $\tau=1000$ (squares), 1200 (circles), 1400 (triangles), 1600 (diamonds), and $1800 \mathrm{~s}$ (crosses). The threshold cross section is $S_{\text {trig }}=12 \mathrm{~km}^{2}$.

$S_{\text {trig }}$ sensitivity experiments, we used $\tau=1200 \mathrm{~s}$ because it better reveals the sensitivity to $S_{d}$ all over the different cases.

Figure 9 shows that the parameterization reacts reasonably to a change in domain size $S_{d}$, favoring triggering on larger domains. The no-triggering probability per unit time $\hat{P}_{\Delta t}$ decreases with $N_{2}$ if a smaller (larger) domain is considered, it is statistically more difficult (easier) to trigger deep convection. For all cases, the sensitivity to $S_{d}$ resembles the sensitivity to $S_{\text {trig }}$ but with the opposite sign: when $S_{d}$ increases, the triggering diurnal cycle is shifted earlier and is more peaked. When the surface domain of reference for the ARM Shallow case is multiplied by a factor $\beta=10$, the probability of triggering having occurred reaches $12 \%$ at the end of the day and $72 \%$ when $\beta=100$. For the BOMEX case, triggering does not happen because $\mathrm{ALE}_{\mathrm{BL} \text {,stat }}<|\mathrm{CIN}|$. To sum up, with this set of parameters, the model has high probabilities of triggering deep convection within a day of simulation, over any land area of $1000 \mathrm{~km} \times$ $1000 \mathrm{~km}$.

This sensitivity to the domain size may be viewed as a "scale dependent" parameterization, which favors triggering when coarse resolutions are used. But, thanks to the stochastic component, this is not the case in a $3 \mathrm{D}$ framework. Here the stochastic component is essential for a "scale aware" triggering parameterization, rather than a scale-dependent one. If a particular domain is considered, whatever the way chosen to divide this domain up, the probability of deep convection being triggered inside it remains unchanged over a large number of realizations. This is because the random number $\mathcal{R}$ is uniformly distributed between 0 and 1 ; then, the no-triggering $\left(\mathcal{R}<\hat{P}_{\Delta t}\right)$ or triggering $\left(\mathcal{R}>\hat{P}_{\Delta t}\right)$ events can be considered as independent from one grid to another. 

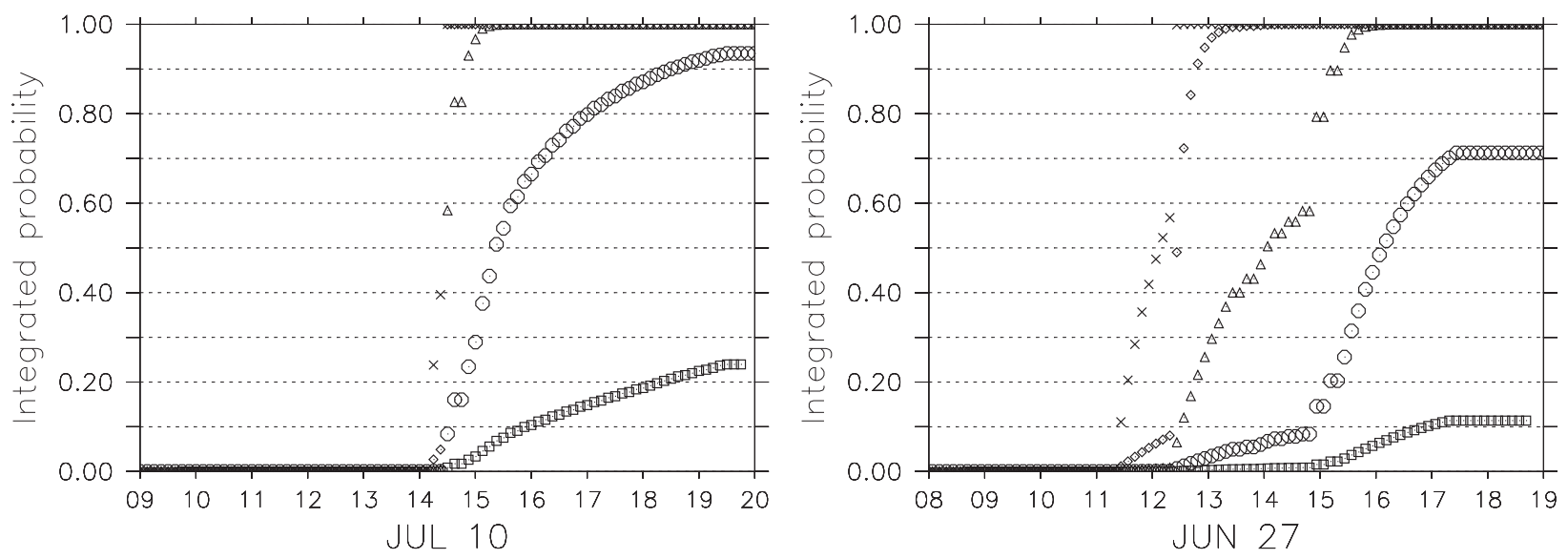

AMMA
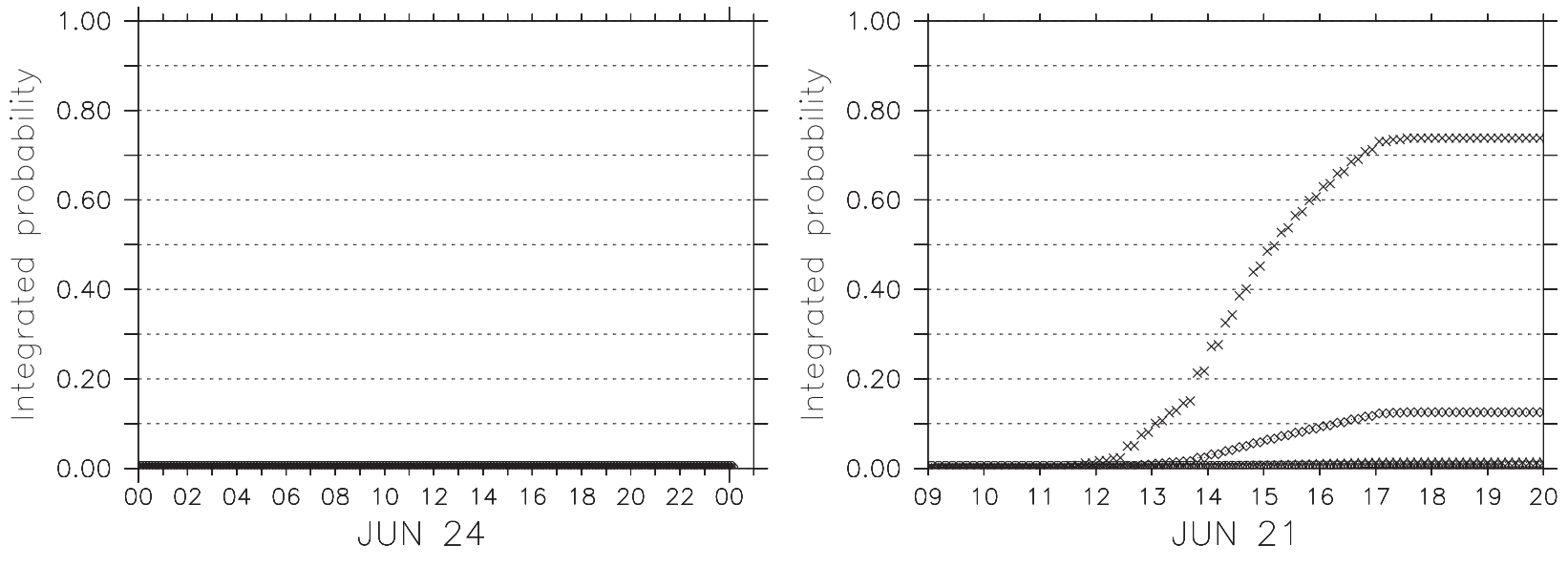

BOMEX

ARM SHALLOW

FIG. 9. As in Fig. 7, but for the domain area of reference (top left) $S_{d, \text { Amma }}=10^{4}$, (top right), (bottom right) $S_{d, \text { Eu }}=6.55 \times 10^{4}$, and (bottom left) $S_{d, \text { Bo }}=2.5 \times 10^{5} \mathrm{~km}^{2}$ multiplied by a factor $\beta=0.01$ (squares), 0.1 (circles), 1 (triangles), 10 (diamonds), and 100 (crosses). The triggering parameters are $S_{\text {trig }}=12 \mathrm{~km}^{2}$ and $\tau=1000 \mathrm{~s}$.

\section{Impact on the deep convection diurnal cycle and day-to-day variability}

In this section, the standard and the new triggering are compared with deep convection switched on. The model was run with $\Delta t=450 \mathrm{~s}$ time step. Each case was run with the standard triggering and the new triggering with the thermal parameters $a=1, b=0.3, \epsilon=0.3$, and $\alpha=0.33$, and the triggering parameters $S_{\text {trig }}=12 \mathrm{~km}^{2}$ and $\tau=$ $1000 \mathrm{~s}$. The domain areas were taken as their reference value (defined in section $3 \mathrm{c}$ ).

\section{a. Impact on deep convection over land: AMMA and ARM Deep cases}

In each of these cases, two "new" runs and a "standard" one are compared. The two stochastic runs correspond to different triggering scenarios, that is, different random samplings. The corresponding simulated diurnal cycles are compared in Figs. 10 and 11.

First, when comparing the new and the standard triggering, one can see that, for both cases, the new parameterization significantly delays deep convection triggering (by $2 \mathrm{~h}$ at least in both cases). This is due to the addition of a supplementary constraint (i.e., the threshold size $S_{\text {trig }}$ ) for the deep convection triggering. Consequently, the precipitation peak is delayed in a similar way. This is the direct consequence of adding a supplementary threshold (a threshold cross section) to the original dynamic threshold (a threshold lifting energy) to enable triggering. Since deep convection tends to inhibit the heating tendencies of boundary layer processes (turbulent diffusion and thermals; see positive $Q_{1, \mathrm{BL}}$ in the right panels of Figs. 10,11) through the subcloud-layer cooling induced by the convective rain 

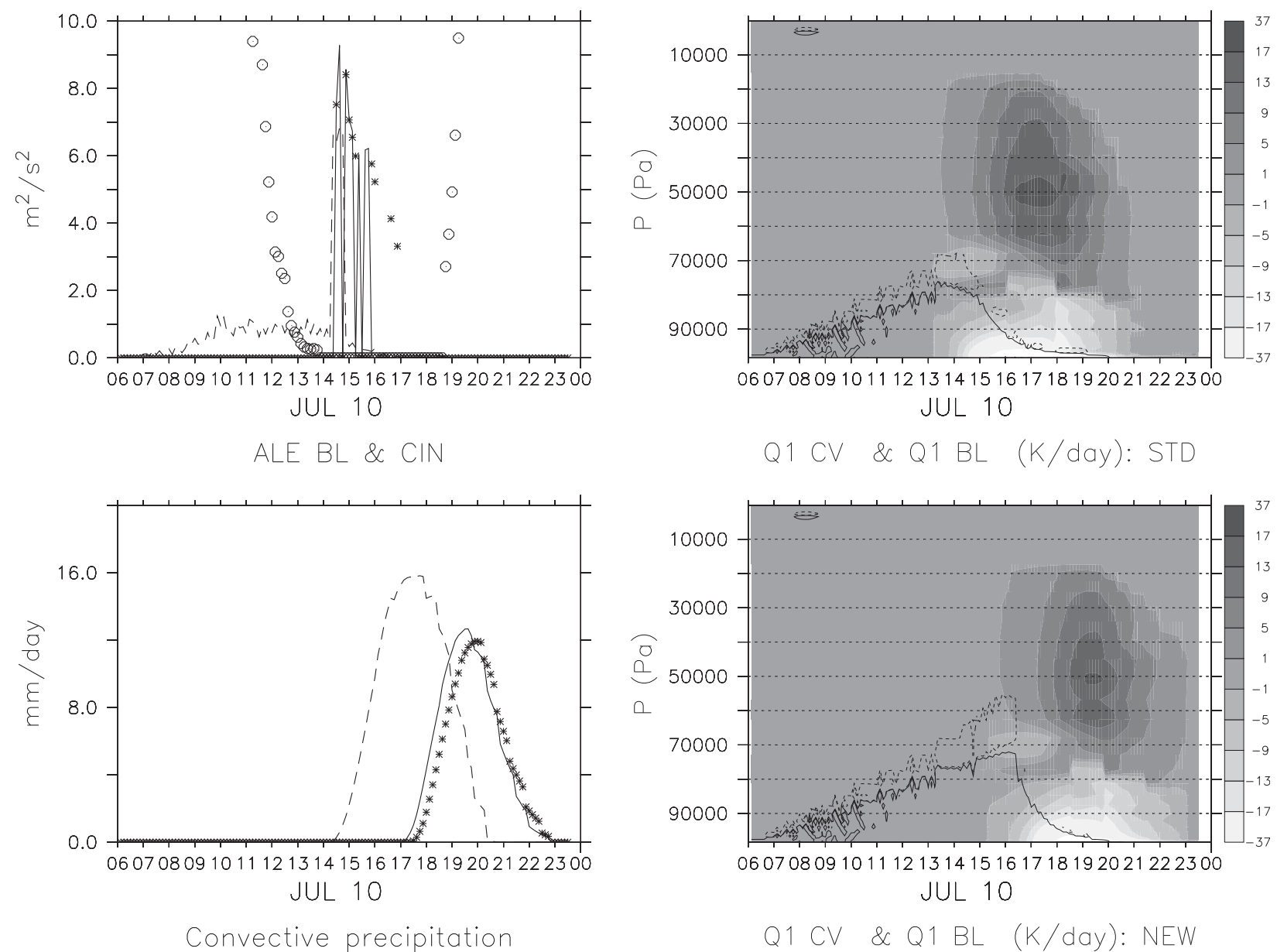

FIG. 10. AMMA case. (top left) CIN (circles; $\mathrm{J} \mathrm{kg}^{-1}$ ) vs $\mathrm{ALE}_{\mathrm{BL}}\left(\mathrm{m}^{2} \mathrm{~s}^{-2}\right.$ or $\mathrm{J} \mathrm{kg}^{-1}$ ) for the standard run (dashed line), the stochastic scenario $1 \mathrm{ALE}_{\mathrm{BL} \text {,eff }}$ (solid line), and the stochastic scenario $2 \mathrm{ALE}_{\mathrm{BL} \text {,eff }}$ (crosses). (bottom left) Convective precipitation (mm day ${ }^{-1}$ ) for the standard run (dashed line), new run 1 (solid line), and new run 2 (crosses). (top right) Deep convection heating rate (K day ${ }^{-1}$ ) $Q_{1, \mathrm{CV}}$ (gray shading) and boundary layer processes heating rate $\left(\mathrm{K} \mathrm{day}^{-1}\right) Q_{1, \mathrm{BL}}$ (thick lines for positive values, from 1 to $40 \mathrm{~K}$ day ${ }^{-1}$, and dashed lines for negative values, from -1 to $-40 \mathrm{~K} \mathrm{day}^{-1}$ ) for the standard run. (bottom right) As in top-right panel, but for new run 1 . The thermal parameters are $a=1, b=0.3, \epsilon=0.3$, and $\alpha=0.33$ and the triggering parameters are $S_{\text {trig }}=12 \mathrm{~km}^{2}$ and $\tau=1000 \mathrm{~s}$. The SCM's deep convection scheme is activated and the time step is $\Delta t=450 \mathrm{~s}$.

re-evaporation (see negative $Q_{1, \mathrm{CV}}$ in the right panels of Figs. 10, 11), boundary layer mixing and deepening last longer in the new run. The boundary layer low-level heating (and drying) and low-tropospheric cooling (and moistening) effects continue later in the afternoon, giving a more continuous transition from the shallow to the deep regime than when the standard triggering is used (see right panels of Figs. 10,11). The negative $Q_{1, \mathrm{BL}}$ zone (dashed contours in Figs. 10, 11), which corresponds to the position of the bulk cumulus simulated by the thermal model, clearly shows that the mean cumulus reaches a more developed stage before triggering in the new run. Looking at Fig. 12, one can see that the boundary layer is significantly drier and deeper at triggering when the triggering is delayed.
Thus, the new triggering allows for a transition phase between shallow and deep regimes, which is partially inhibited by the deep convection scheme in the standard parameterization. This more gradual transition is more in phase with LES and observational data (see Lothon et al. 2011; Couvreux et al. 2012; Guichard et al. 2004). It should be noted that regarding the diurnal cycle of precipitation on the ARM Deep case, both the standard and new runs fall within the range of the CRM results presented in Guichard et al. (2004). As in the CRMs, the later the triggering, the weaker is the intensity of precipitation.

Figures 10 and 11 show that there is a 2 -h time lag between deep convection triggering and rainfall. It is caused by the existence of a convective adjustment time 

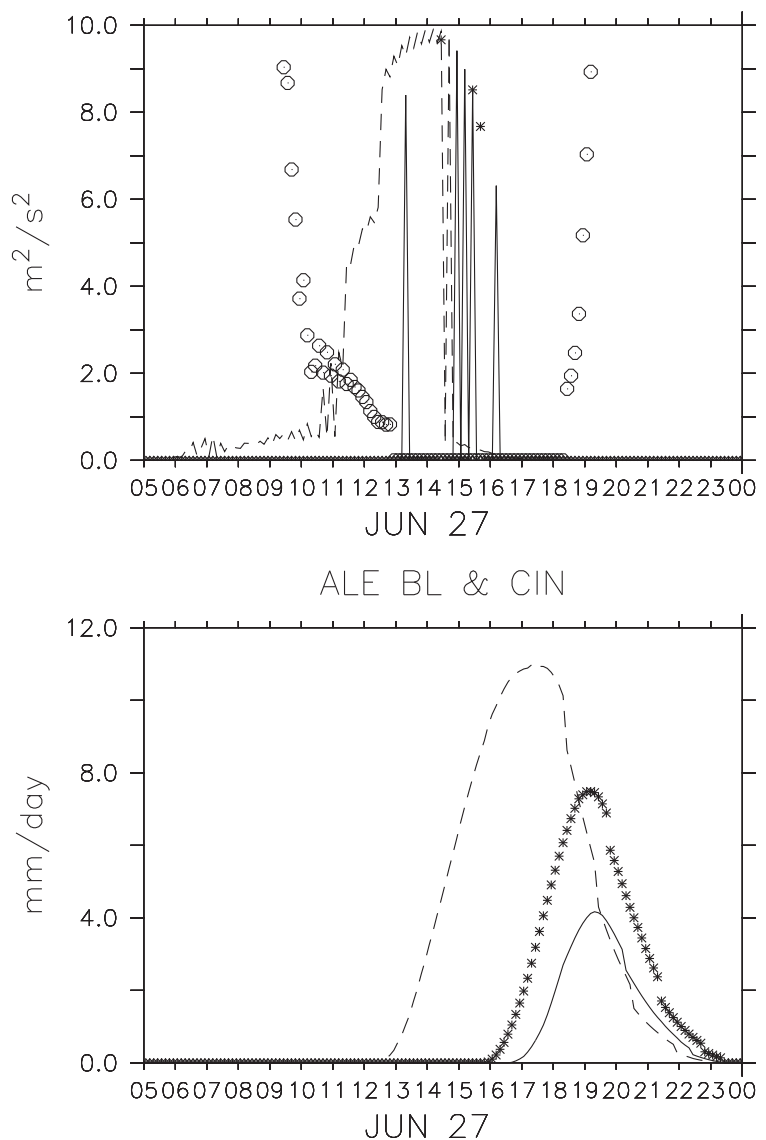

Convective precipitation

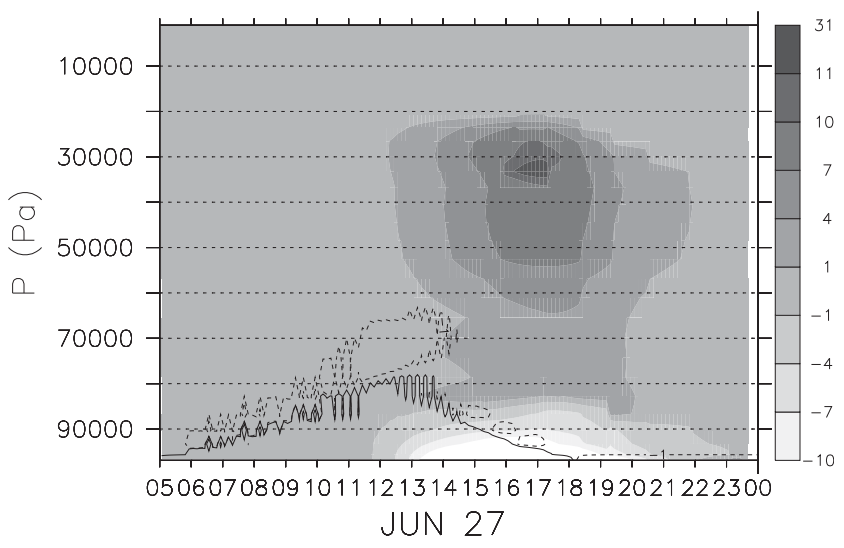

Q1 CV \& Q1 BL (K/day): STD

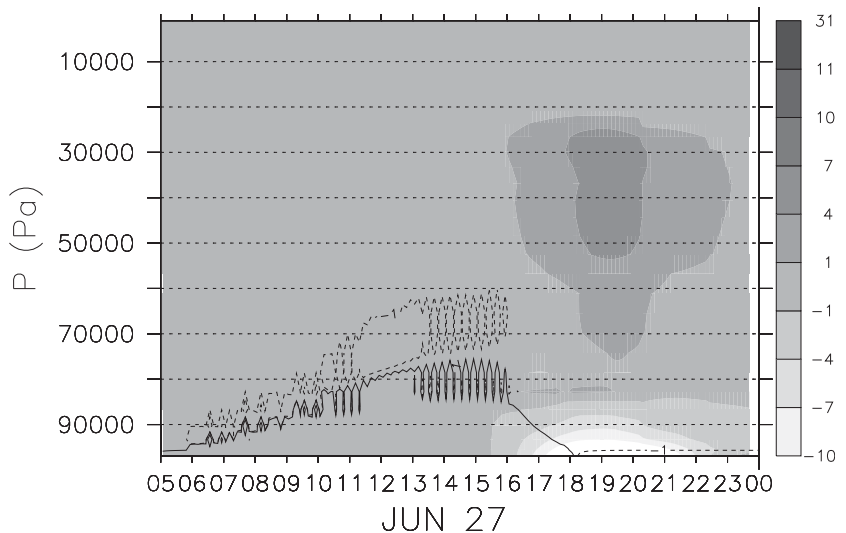

Q1 CV \& Q1 BL (K/day): NEW

FIG. 11. As in Fig. 10, but for the ARM Deep case.

parameter, which governs the time scale of response of deep convection to large-scale perturbations. This parameter is one of the so-called tuning parameters (see Hourdin et al. 2013) and is currently set to $8000 \mathrm{~s}$ in the standard version of LMDZ5B.

We now compare two different scenarios (see dashed lines and crosses in bottom-left panels in Figs. 10, 11) given by the new runs and corresponding to two distinct random realizations $\mathcal{R}$. For the AMMA case, both runs trigger around $1430 \mathrm{LT}$ (Fig. 10). As shown in Fig. 7, the integrated triggering probability [Eq. (19)] increases dramatically at that time and most of the triggering times are concentrated in this short period. On the other hand, for the ARM Deep case (Fig. 11), the triggering scenarios differ by several hours and the probability of triggering is more spread over the afternoon (see Fig. 7). Thus, the stochastic component increases the intraday variability by making the model able to trigger deep convection at very different times of the day under similar conditions.

Concerning deep convection intensity, in the AMMA case (Fig. 10), both standard and new runs exhibit similar diabatic heating rates $Q_{1, \mathrm{CV}}$. However, for ARM Deep (Fig. 11), convection intensity is significantly weaker in the new case. Actually, the fact that deep convection arises later in the new parameterization has two opposite effects. First, the triggering occurs in a deeper and drier boundary layer and a moister, lower free troposphere (see Fig. 12) because boundary layer processes last longer. The drier and deeper the boundary layer is, the higher and colder the cold pools are. In LMDZ, such developed cold pools should a priori increase the cloud-base mass flux through the ALP closure [see Eq. (3)], thus leading to stronger deep convection. However, when triggering occurs later, the CIN returned by the SCM might also be higher (see Fig. 5), and the present closure [Eq. (3)] should give a lower cloud-base mass flux. In the AMMA case, those opposite effects tend to compensate one another, while in the ARM Deep case, the second effect dominates. Thus, the time of triggering has a strong impact on deep convection intensity. 


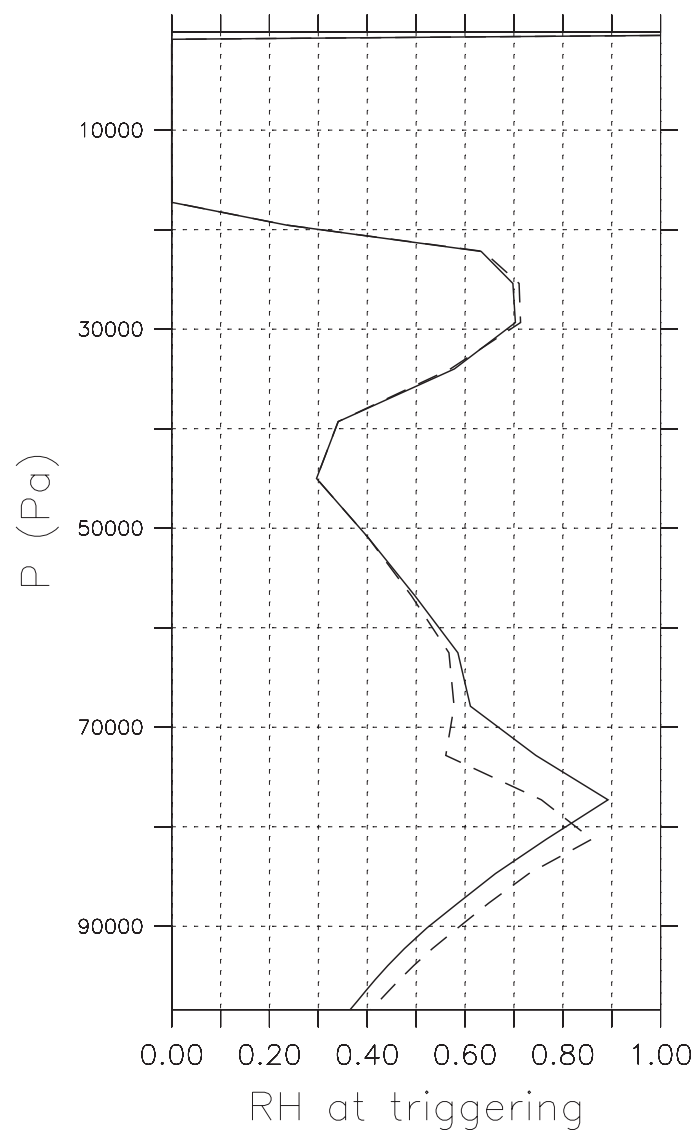

AMMA

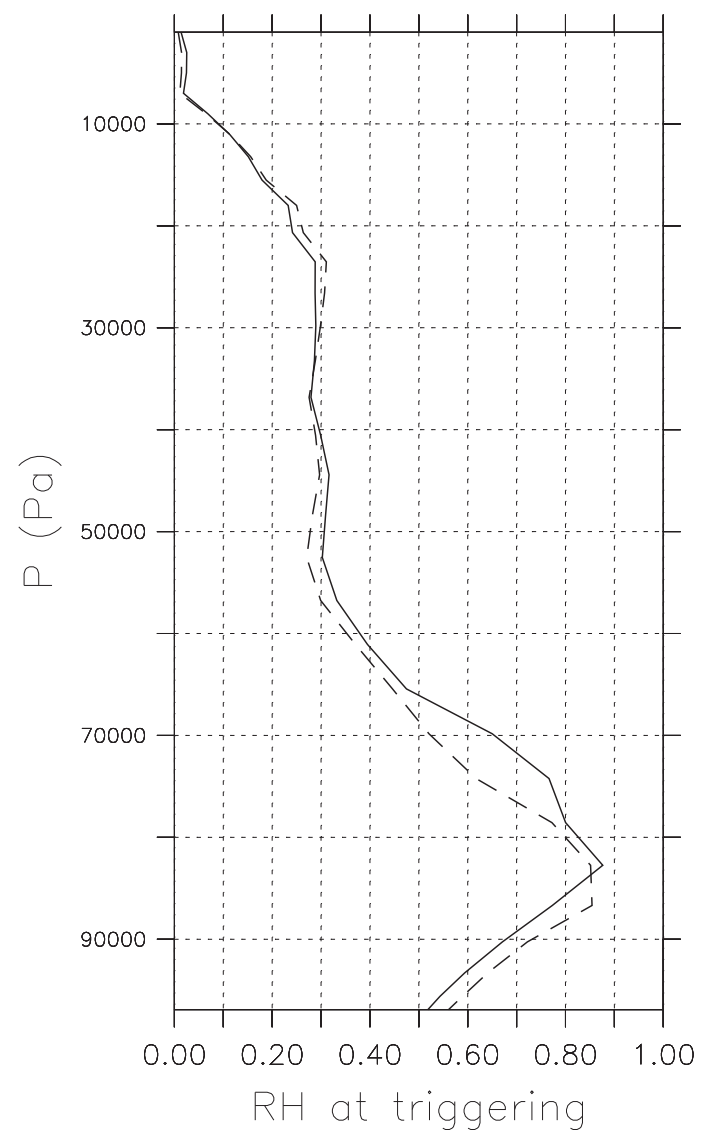

ARM DFEP

FIG. 12. Relative humidity profiles at triggering for the standard (dashed lines) and the new (solid lines) runs extracted from the SCM for (left) AMMA case and (right) ARM Deep case. The thermal parameters are $a=1, b=$ $0.3, \epsilon=0.3$, and $\alpha=0.33$ and the triggering parameters are $S_{\text {trig }}=12 \mathrm{~km}^{2}$ and $\tau=1000 \mathrm{~s}$. The SCM's deep convection scheme is activated and the time step is $\Delta t=450 \mathrm{~s}$.

\section{b. Impact on the shallow convection: BOMEX and ARM Shallow cases}

According to the sensitivity study plotted in Fig. 7, the new triggering cancels deep convection onset in the BOMEX case and shows only slight probability in ARM Shallow with the current set of parameters. Therefore, in Figs. 13 and 14, only one "no-trigger scenario" (unlike Figs. 10,11) is considered in the new runs as it is representative of the great majority of the possibilities. It is then compared with the standard triggering.

On the one hand, the standard runs both trigger deep convection, and some precipitation is even simulated in BOMEX. On the other hand, the new runs do not trigger and $A L E_{B L, \text { eff }}$ remains 0 . Results from the new runs are then consistent with LES and observational data that are given in Siebesma et al. (2003) and Brown et al. (2002) for the BOMEX and ARM Shallow cases, respectively. Indeed, neither precipitation nor deep convective clouds have been reported in both of these cases, regarding the reference papers cited above.

In the BOMEX case, the absence of triggering is explained solely by the fact that the statistical $\mathrm{ALE}_{\mathrm{BL} \text {,stat }}$ stays below CIN. In the ARM Shallow case, both

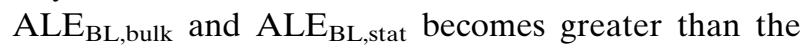
CIN at approximately the same time (around 1130 LT) but, in the new run, the cloud depth and LCL altitude are too small to make sufficiently wide thermals [Eq. (14)] and to make them decrease the no-triggering probability $\left[\hat{P}_{\Delta t}\right.$, Eq. (8)] significantly. The variable $\hat{P}_{\Delta t}$ stays very close to 1 (not shown) throughout the day, which prevents triggering. Thus, in both new runs, the boundary layer is responsible for $100 \%$ of the mixing processes and the cumulus cloud development is not altered by the counter effect of deep convection. As already stressed in the previous subsection, this favors the presence of a drier boundary layer together with a moister, lower free troposphere (not shown). 

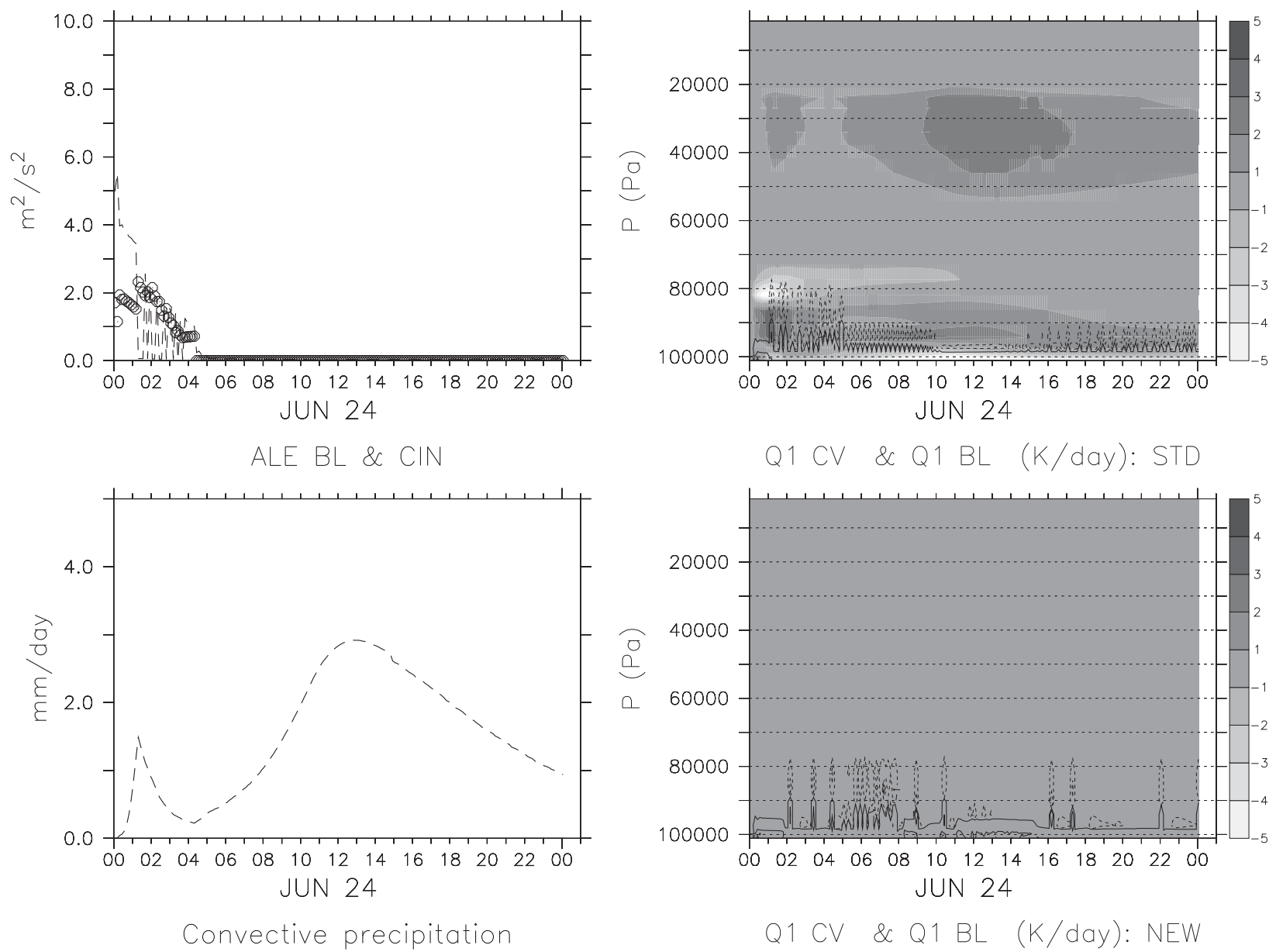

FIG. 13. BOMEX case. (top left) CIN (circles; $\mathrm{J} \mathrm{kg}^{-1}$ ) vs standard ALE $\mathrm{BL}$ (dashed line; $\mathrm{m}^{2} \mathrm{~s}^{-2}$ or J kg ${ }^{-1}$ ) and the new ALE line). (bottom left) Convective precipitation $\left(\mathrm{mm} \mathrm{day}^{-1}\right)$ for the standard (dashed line) and the new (solid line) runs; in both panels the solid line is on top of the horizontal axis. (right) As in Fig. 10, but for BOMEX.

\section{c. Preliminary results from $3 D$ runs}

To assess the ability of the new triggering parameterization to perform reasonably well in a large range of conditions, we implemented it in the LMDZ5 GCM. The LMDZ model was run for several decades with a $96 \times 95 \times 39$ grid and forced with climatological SSTs. We must stress that the results presented in this subsection are preliminary. Note that simulations have been retuned in order to conserve a correct energy balance at the top of the atmosphere [see tuning methodology used in LMDZ5 in Hourdin et al. (2013)]. Here we compare some LMDZ outputs obtained using either the standard triggering or the new (stochastic) triggering.

\section{1) SPATIAL VARIABILITY OF DEEP CONVECTION}

Cold pools are associated with deep convection in the model. Hence, their average map of frequency of occurrence (given in Fig. 15) can be associated with the deep convection mean spatial distribution. From Fig. 15, we learn that the new triggering has two notable effects on the deep convection spatial distribution.

First, on average, the new triggering decreases the deep convection frequency of occurrence all around the globe. In convective zones, such as the eastern Pacific and Atlantic Oceans, or even the Amazon basin, deep convection occurs less frequently. The area extent of the West African monsoon is also slightly reduced on its northern and eastern boundaries. In subsiding zones, deep convection is also cancelled over much larger areas in the new run than in the standard run. This is particularly true over the eastern tropical oceans, but it is also prominent when the northern Atlantic and Pacific Oceans are observed. This makes the ITCZ appear more distinctly in the new run.

Second, the spatial variability of deep convection is increased. When looking at the warm pool area, for instance, one can clearly see that the pattern is patchier when the new triggering is used. 

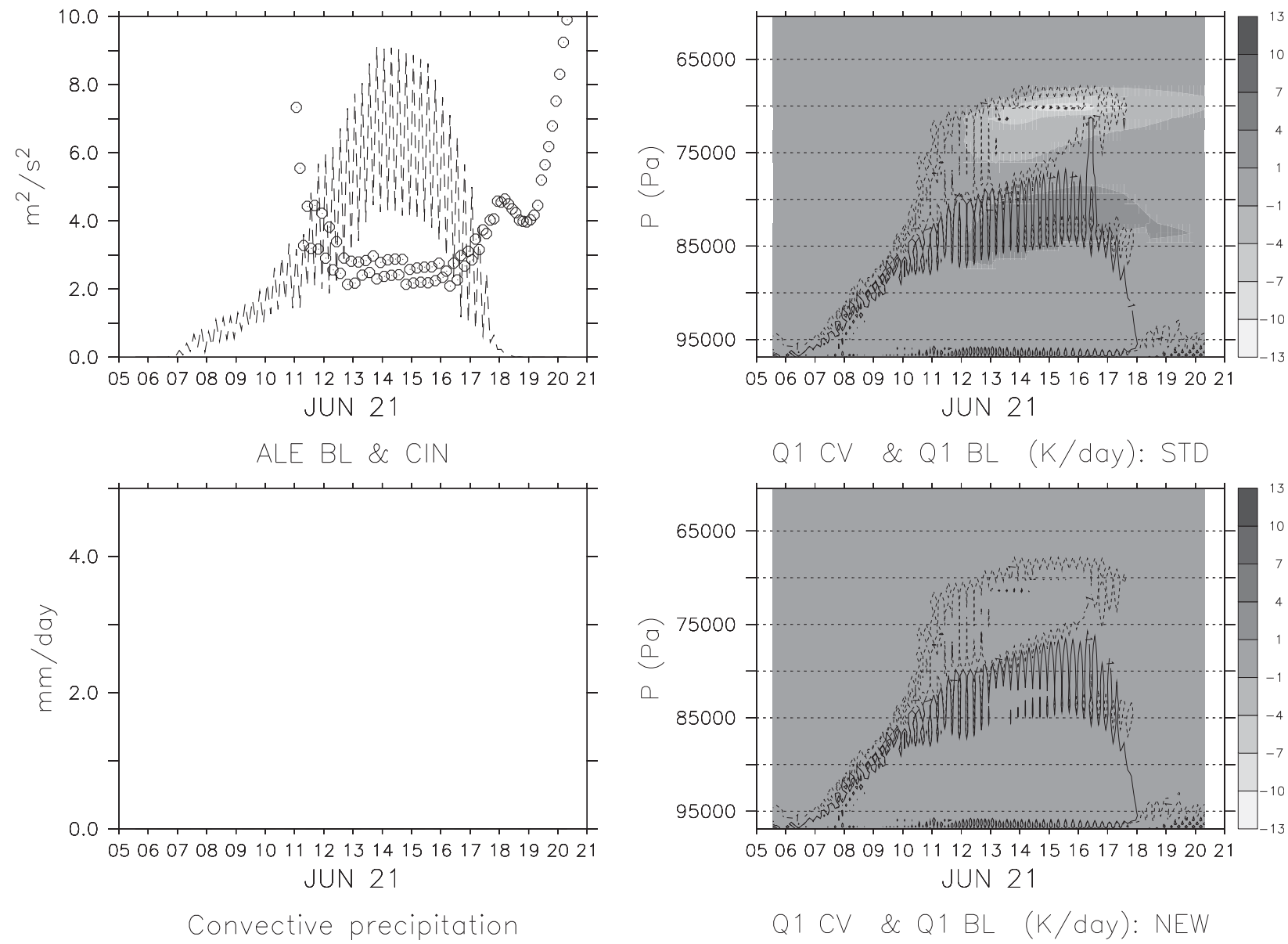

FIG. 14. As in Fig. 13, but for the ARM Shallow case.

Those results are presumably due to both (i) the new computation of $\mathrm{ALE}_{\mathrm{BL}}$ and (ii) the addition of a supplementary threshold $S_{\text {trig, }}$, which are included in the new triggering parameterization. Thus, the new triggering makes deep convection sparser in space and time, decreasing its frequency of occurrence and increasing its spatial variability. We also recall that this aspect is consistent with the episodic nature of deep convection triggering that was asserted in Part I.

\section{2) LOW-LEVEL CLOUDINESS}

Figure 16 shows the average low-level cloudiness for the month of July, returned by the standard and the new triggering parameterization. From Fig. 16, it is clear that the new triggering increases the low-level cloudiness in the tropics. Differences are particularly large in the winter hemisphere, where the subsiding branch of the Hadley cell is the strongest. This increase of the lowlevel cloud cover is consistent with the results presented in Figs. 13 and 15. In fact, since deep convection is cancelled over subsiding zones, boundary layer turbulence processes are no longer affected by the low-level cooling due to precipitation and cold pools. Thus, without the counter effect of deep convection, boundary layer eddies are more efficient at transporting heat and moisture up to the lifting condensation level to create stratocumulus and cumulus clouds. Given that LMDZ5B tends to underestimate low-level cloudiness in subsiding zones, this aspect could be of critical importance.

\section{3) TEMPORAL VARIABILITY OF DEEP CONVECTION}

Figure 17 displays the convective precipitation time series simulated over a grid point located in the Sahel (nearly over Niamey) during a particular month of July of the long-term simulation (i.e., during the monsoon). The new triggering significantly increases the day-to-day variability of deep convection. Figure 17 shows that precipitation occurs almost every day in the standard run, whereas it rains approximately every other day in the new run. This day-to-day variability is directly related to the introduction of a stochastic term in the triggering 

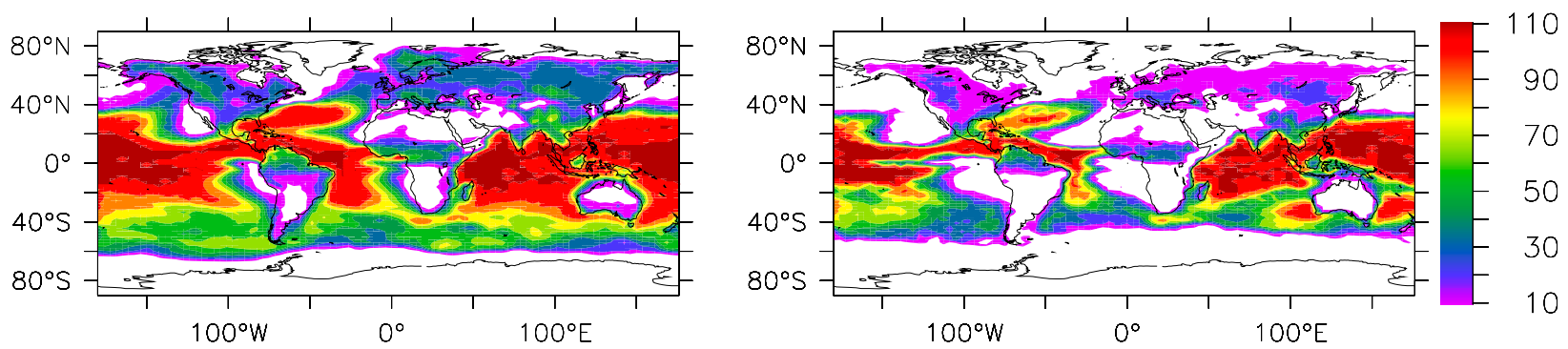

FIG. 15. Map of the cold pool frequency of occurrence (\%) for the month of July averaged over $10 \mathrm{yr}$ of simulation. This map corresponds to the fraction of time when cold pools are present in each GCM grid over the month of July. LMDZ (left) with standard and (right) new triggering. The thermal parameters are $a=1, b=0.3, \epsilon=0.3$, and $\alpha=0.33$ and the triggering parameters are $S_{\text {trig }}=12 \mathrm{~km}^{2}$ and $\tau=$ $600 \mathrm{~s}$. The GCM's time step is $\Delta t=450 \mathrm{~s}$.

parameterization. Even if the large-scale conditions are favorable, the new parameterization does not trigger unless a random number exceeds a certain value, yielding a "stochastic variability." The consequence is that triggering is never guaranteed, even if thermals overcome CIN. This is of particular interest over semiarid zonessometimes classified as "marginal zones" (Charney et al. 1977) or "hot spots" (Koster et al. 2004) - in which the day-to-day variability is a major climatic component. Then, these preliminary results suggest that the stochastic triggering parameterization could also improve the model's representation of the day-to-day variability of deep convection in the tropics.

\section{Conclusions}

The parameterization described in this paper is derived from the analysis of LES data made in Part I of this study. The study of the statistical properties of the thermal spectrum revealed great advantages. It (i) allowed hypotheses to be states for building the thermal distribution parameterization and (ii) suggested the need for a supplementary, stochastic threshold governing the onset of deep convection. This resulted in a new formulation of the transition from shallow to deep convection, which includes a spectral representation of the cloudy thermals and a stochastic triggering of deep convection. From that, a new parameterization of deep convection triggering by boundary layer thermals was proposed for the LMD's GCM (LMDZ5B). Among other considerations, we assume a linear relationship between the mean cross section of the thermals, the LCL altitude and the cloud depth extracted from the thermal model [developed by Rio and Hourdin (2008)]. This parameterization thus includes a computation of the thermal cross-sectional spectrum and a computation of a no-triggering probability, which, when exceeded by a random sample, determines whether triggering occurs or not. It accounts for six parameters, four of which $(a, b, \epsilon$, and $\alpha)$ are related to the cloudy thermals spectrum computation (mean cross section $S_{2}$ and population $N_{2}$ ), and the others $\left(S_{\text {trig }}\right.$ and $\left.\tau\right)$ are related to the notriggering probability computation $\left(\hat{P}_{\Delta t}\right)$.

A sensitivity study was made over the threshold cross section $S_{\text {trig }}$ and the decorrelation time $\tau$ in order to explore some general features of the new stochastic triggering parameterization.

(i) Over drier surfaces, the transition regime between shallow and deep convection is shorter than over wet surfaces (where the cloud base is low).

(ii) The new triggering is still largely controlled by large-scale and surface conditions but allows the presence of an intermediate stage between shallow and deep regimes, in which stochasticity can deeply affect the diurnal cycle scenario via the local time of triggering.

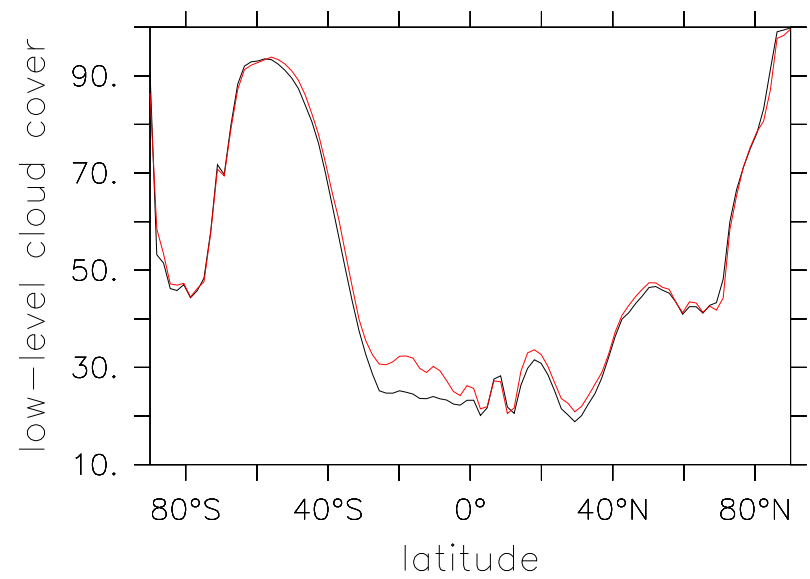

FIG. 16. Zonal mean of the low-level cloudiness for the month of July, when averaged over $10 \mathrm{yr}$ of simulation. Black line is LMDZ with standard and red line with new triggering. The thermal parameters are $a=1, b=0.3, \epsilon=0.3$, and $\alpha=0.33$ and the triggering parameters are $S_{\text {trig }}=12 \mathrm{~km}^{2}$ and $\tau=600 \mathrm{~s}$. The GCM's time step is $\Delta t=450 \mathrm{~s}$ 


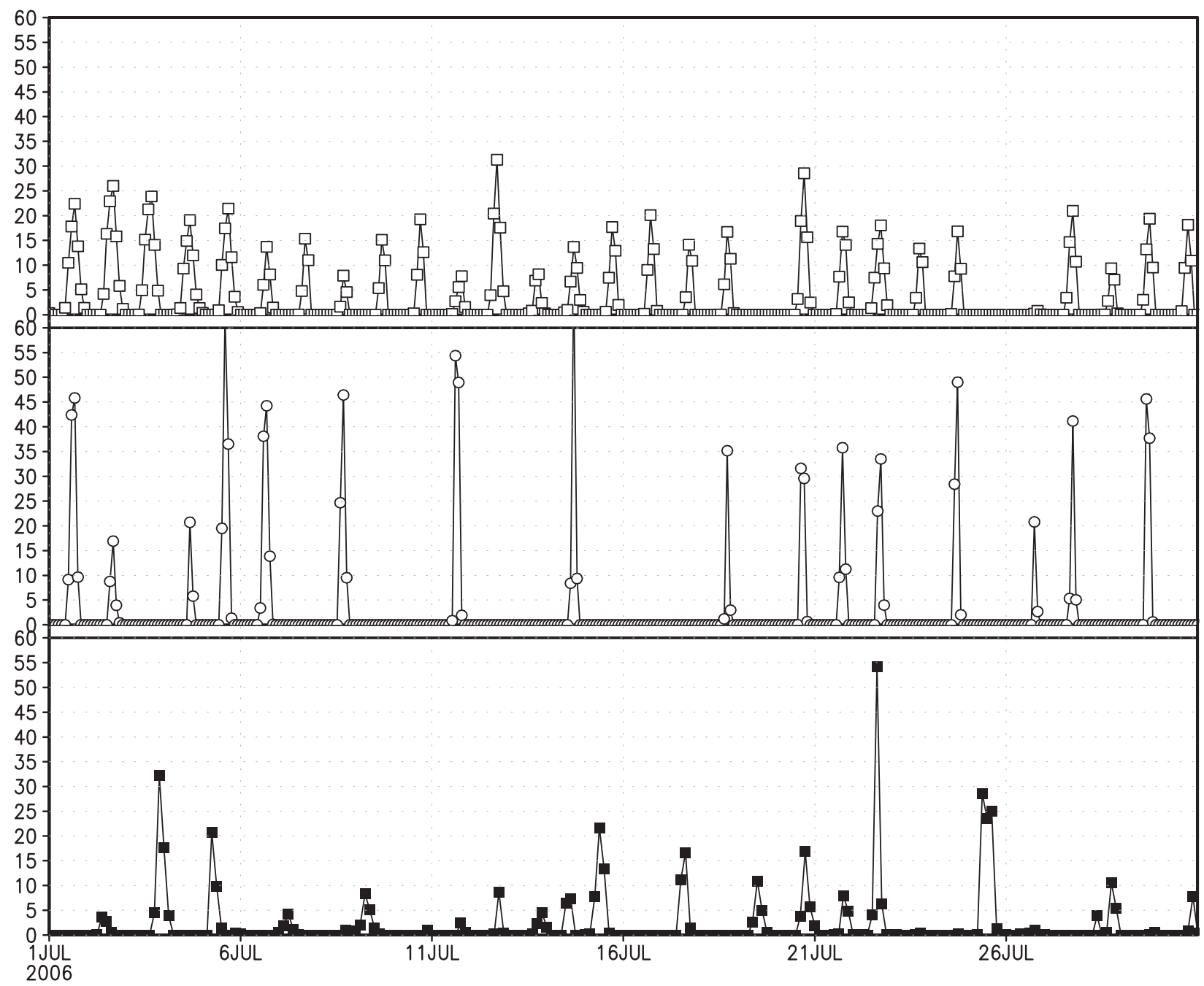

FIG. 17. Time series of convective precipitation $\left(\mathrm{mm} \mathrm{h}^{-1}\right)$ over Niamey $\left(0^{\circ}, 12^{\circ} \mathrm{N}\right.$, Niger) for the month of July. LMDZ (top) with standard and (middle) new triggering, and (bottom) Tropical Rainfall Measuring Mission satellite data. The thermal parameters are $a=1$, $b=0.3, \epsilon=0.3$, and $\alpha=0.33$ and the triggering parameters are $S_{\text {trig }}=12 \mathrm{~km}^{2}$ and $\tau=600 \mathrm{~s}$. The GCM's time step is $\Delta t=450 \mathrm{~s}$.

(iii) The stochastic component ensures the scale awareness of the parameterization. It is then sensitive to the domain area considered (in a 1D framework), but insensitive to horizontal resolution (in a 3D framework). The scale-aware nature of the new triggering gives significant added value to the GCM.

Triggering appears to be a scarce process, which has a certain probability of occurring given the large-scale and the surface conditions. In other words, even in favorable conditions, deep convection may not be triggered. By similar reasoning, it can potentially be triggered in a relatively unfavorable environment.

Results from 1D case studies and 3D long-term simulations show that the new triggering is not only better at capturing the deep convection diurnal cycle (see previous subsection), but also at deciding when and where deep convection should or should not be active.

First, the new $\mathrm{ALE}_{\mathrm{BL}}$ computation seems to prevent oceanic subsiding zones from deep convection; this mostly comes from the choice to take a reference velocity at cloud base instead of considering the maximum velocity over the thermal profile. Then, the multistep triggering tends to delay deep convection onset, allowing for a realistic transition stage. Finally, the stochastic component acts to increase the system's variability (especially via the intraday and day-to-day variability).

This results in a more realistic representation of the spatial and temporal variability of moist convection: the stochastic triggering parameterization makes it possible to obtain a better alternation between dry and rainy days and subsiding and ascending zones. 
Ultimately, these encouraging results give us hope that the scale-aware and stochastic parameterization proposed here could open the way to the better capture of the dominant modes of intraseasonal variability of moist convection in the tropics, which is still poorly represented in most current GCMs.

Acknowledgments. The research leading to these results has been supported by both the French Department of Teaching and Research and the European Union, Seventh Framework Program (FP7/2011-2015) under Grant Agreement n282672. The authors thank Francoise Guichard and Jean-Philippe Lafore, from Météo-France (Toulouse), for their very helpful comments and for the numerous and enlightening discussions we had about deep convection issues. The authors also thank Frédéric Hourdin, Marie-Pierre Lefèbvre, and Abderrahmane Idelkadi for their help regarding the LMDZ simulations. Finally, they thank the three anonymous reviewers, whose meticulous analysis and enlightening suggestions made it possible to improve the quality of the manuscript.

\section{REFERENCES}

Bechtold, P., J. Chaboureau, A. Beljaars, A. Betts, M. Köhler, M. Miller, and J. Redelsperger, 2004: The simulation of the diurnal cycle of convective precipitation over land in a global model. Quart. J. Roy. Meteor. Soc., 130, 3119-3137.

Bony, S., and K. Emanuel, 2001: A parameterization of the cloudiness associated with cumulus convection; Evaluation using TOGA COARE data. J. Atmos. Sci., 58, 31583183.

Brown, A., and Coauthors, 2002: Large-eddy simulation of the diurnal cycle of shallow cumulus convection over land. Quart. J. Roy. Meteor. Soc., 128, 1075-1093.

Charney, J., W. Quirk, S. Chow, and J. Kornfield, 1977: A comparative study of the effects of albedo change on drought in semi-arid regions. J. Atmos. Sci., 34, 1366-1385.

Couvreux, F., C. Rio, F. Guichard, M. Lothon, G. Canut, D. Bouniol, and A. Gounou, 2012: Initiation of daytime local convection in a semi-arid region analysed with high-resolution simulations and AMMA observations. Quart. J. Roy. Meteor. Soc., 138, 56-71.

Emanuel, K., 1991: A scheme for representing cumulus convection in large-scale models. J. Atmos. Sci., 48, 2313-2335.

Grandpeix, J., and J. Lafore, 2010: A density current parameterization coupled with Emanuel's convection scheme. Part I: The models. J. Atmos. Sci., 67, 881-897.
_ _ V. Phillips, and R. Tailleux, 2004: Improved mixing representation in Emanuel's convection scheme. Quart. J. Roy. Meteor. Soc., 130, 3207-3222.

Guichard, F., and Coauthors, 2004: Modelling the diurnal cycle of deep precipitating convection over land with cloud-resolving models and single-column models. Quart. J. Roy. Meteor. Soc., 130, 3139-3172.

Hohenegger, C., and B. Stevens, 2013: Preconditioning deep convection with cumulus congestus. J. Atmos. Sci., 70, 448464.

Holland, J., 1970: Preliminary report on the bomex sea-air interaction program. Bull. Amer. Meteor. Soc., 51, 815-816.

Hourdin, F., and Coauthors, 2006: The LMDZ4 general circulation model: Climate performance and sensitivity to parametrized physics with emphasis on tropical convection. Climate Dyn., 27, 787-813.

- and Coauthors, 2013: LMDZ5B: The atmospheric component of the IPSL climate model with revisited parameterizations for clouds and convection. Climate Dyn., 40, 2193-2222, doi:10.1007/s00382-012-1343-y.

Koster, R., and Coauthors, 2004: Regions of strong coupling between soil moisture and precipitation. Science, 305, 11381140.

Lothon, M., B. Campistron, M. Chong, F. Couvreux, F. Guichard, C. Rio, and E. Williams, 2011: Life cycle of a mesoscale circular gust front observed by a c-band doppler radar in west africa. Mon. Wea. Rev., 139, 1370-1388.

Mellor, G., and T. Yamada, 1974: A hierarchy of turbulence closure models for planetary boundary layers. J. Atmos. Sci., 31, 1791-1806.

Rio, C., and F. Hourdin, 2008: A thermal plume model for the convective boundary layer: Representation of cumulus clouds. J. Atmos. Sci., 65, 407-425.

$\_, \ldots$, J. Grandpeix, and J. Lafore, 2009: Shifting the diurnal cycle of parameterized deep convection over land. Geophys. Res. Lett., 36, L07809, doi:10.1029/2008GL036779.

,$- \ldots$, F. Couvreux, and A. Jam, 2010: Resolved versus parametrized boundary-layer plumes. Part II: Continuous formulations of mixing rates for mass-flux schemes. Bound.-Layer Meteor., 135, 469-483.

— , and Coauthors, 2013: Control of deep convection by subcloud lifting processes: The alp closure in the LMDZ5B general circulation model. Climate Dyn., 40, 2271-2292, doi:10.1007/ s00382-012-1506-x.

Rochetin, N., F. Couvreux, J.-Y. Grandpeix, and C. Rio, 2014: Deep convection triggering by boundary layer thermals. Part I: LES analysis and stochastic triggering formulation. J. Atmos. Sci., 71, 496-514.

Siebesma, A. P., and Coauthors, 2003: A large eddy simulation intercomparison study of shallow cumulus convection. $J$. Atmos. Sci., 60, 1201-1219.

Yang, G., and J. Slingo, 2001: The diurnal cycle in the tropics. Mon. Wea. Rev., 129, 784-801. 